\title{
QUANTUM LEFSCHETZ HYPERPLANE THEOREM
}

\author{
Y.-P. LEE
}

\begin{abstract}
The mirror theorem is generalized to any smooth projective variety $X$. That is, a fundamental relation between the Gromov-Witten invariants of $X$ and Gromov-Witten invariants of complete intersections $Y$ in $X$ is established.
\end{abstract}

\section{Notation.}

- Our notations follow those in [8] unless otherwise mentioned.

- The (co)homology/Chow groups are over $\mathbb{Q}$ and all varieties (schemes, stacks) are over ground field $\mathbb{C}$.

- $X$ is a smooth projective variety. $i: X \hookrightarrow P:=\prod_{i=1}^{N} \mathbb{P}^{r_{i}}$ is and embedding such that $i^{*}: N S\left(\prod \mathbb{P}^{r_{i}}\right) \rightarrow N S(X)$ is an isomorphism, where $N S(X)=$ $Z^{1}(X) / \sim_{\text {hom }}$ is the Neron-Severi group.

- Let $p_{i}:=i^{*}\left(h_{i}\right)$, where $h_{i}$ is the hyperplane class of $\mathbb{P}^{r_{i}}$.

- For each $\beta \in H_{2}(X) d(\beta):=i_{*}(\beta) \in H_{2}(P)$.

- $\pi_{k}: \bar{M}_{0, n+1}(Y, \beta) \rightarrow \bar{M}_{0, n}(Y, \beta)$ is the forgetful morphism which forgets the $k$-th marking. $\pi:=\pi_{n+1}$.

- ev : $\bar{M}_{0, n}(X, \beta) \rightarrow(X)^{n}$ is the evaluation morphism.

- $p_{k}: Y_{1} \times{ }_{Z} Y_{2} \rightarrow Y_{k}$ are the projection morphisms.

- $\bar{G}_{0, n}(X, \beta):=\bar{M}_{0, n}\left(X \times \mathbb{P}^{1},(\beta, 1)\right)$ is called $n$-pointed graph space of $X$.

- For a convex vector bundle $E$ on $X$, define a vector bundle $E_{\beta}:=\pi^{*}\left(R^{0} \pi_{*} \mathrm{ev}^{*} E\right)$ on $\bar{M}_{0,1}(X, \beta)$. If $E$ is concave, $E_{\beta}:=\pi^{*}\left(R^{1} \pi_{*} \mathrm{ev}^{*} E\right)$. Similar definition on $\bar{G}_{0, n}(X, \beta): E_{\beta}^{G}:=R^{j} \pi_{*} \mathrm{ev}^{*} E, j=0$ for convex $E$ and $j=1$ for concave $E$.

- A stable map is denoted by a quadruple $(C, x, f: C \rightarrow X, \beta)$, where $x=$ $\left(x_{1}, \cdots, x_{n}\right)$ are the marked points, $\beta$ is the degree of the map $f$.

- Let $f: V \rightarrow M$ be a morphism with $M$ smooth, then $f_{*}^{\text {vir }}: H^{*}(V) \rightarrow H^{*}(M)$ is defined to be

$$
f_{*}^{\mathrm{vir}}(\alpha):=\mathrm{PD} \circ f_{*}\left(\alpha \cap[V]^{\mathrm{vir}}\right),
$$

where PD $: H_{*}(M) \rightarrow H^{*}(M)$ is the Poincaré duality map and $f_{*}: H_{*}(V) \rightarrow$ $H_{*}(M)$ is the push-forward of cycles. Note that $f_{*}$ is also used for pushforward of cohomology classes when $V$ is also smooth. There should be no confusion.

- $\psi:=c_{1}\left(\mathcal{L}_{1}\right)$ is the universal cotangent class. Namely $\mathcal{L}=x_{1}^{*}\left(\omega_{C / M}\right)$, where $\omega_{C / M}$ is the relative dualizing sheaf of the universal curve $C \rightarrow \bar{M}_{0,1}(X, \beta)$ and $x_{1}$ is the marked point.

- All objects considered on graph space and on linear sigma model $\mathbb{P}_{d}^{r}:=$ $\mathbb{P}^{(r+1) d+r}$ are $\mathbb{C}^{*}$-equivariant.

1991 Mathematics Subject Classification. Primary: 14N35.

Research partially supported by NSF grant. 
- We have summarized the most useful notations (other than those listed above) in the commutative diagrams (10) and (11).

\section{INTRODUCTION}

1.1. Statement of the main results. Let $X$ be a smooth projective variety. Let $T_{k}$ be a basis of $H^{*}(X), T_{0}=1, T_{i}=p_{i}, i=1, \cdots N$ and let $t_{k}$ be the dual coordinates of $T_{k}$. Let $g_{m k}:=\int_{X} T_{m} \cup T_{k}$ the intersection pairing and $\left(g^{m k}\right)$ is the inverse matrix of $\left(g_{m k}\right)$. Let $E:=\left(\oplus_{j} L_{j}\right)$ be a vector bundle on $X$, where $L_{j}$ are pull-backs of convex/concave (see below) line bundles on $P$. Define the generating function of genus zero one-point gravitational Gromov-Witten invariants on $X$ to be

$$
\begin{aligned}
J_{X}(q, \hbar) & :=e^{\frac{1}{\hbar}\left(t_{0}+\sum_{i} p_{i} t_{i}\right)} \sum_{\beta} q^{\beta} J_{X}(\beta) \\
& :=e^{\frac{1}{\hbar}\left(t_{0}+\sum_{i} p_{i} t_{i}\right)} \sum_{\beta} q^{\beta} \mathrm{ev}_{*}^{\mathrm{vir}} \frac{1}{\hbar(\hbar-\psi)} \\
& =e^{\frac{1}{\hbar}\left(t_{0}+\sum_{i} p_{i} t_{i}\right)} \sum_{m k} T_{m} g^{m k} \sum_{\beta} q^{\beta} \int_{\left[M_{0,1}(X, \beta)\right]^{\mathrm{vir}}} \operatorname{ev}^{*}\left(T_{k}\right) \frac{1}{\hbar(\hbar-\psi)}
\end{aligned}
$$

and for $E \rightarrow X$ :

$$
J_{X}^{E}(q, \hbar):=e^{\frac{1}{\hbar}\left(t_{0}+\sum_{i} p_{i} t_{i}\right)} \sum_{\beta} q^{\beta} J_{X}^{E}(\beta):=e^{\frac{1}{\hbar}\left(t_{0}+\sum_{i} p_{i} t_{i}\right)} \operatorname{ev}_{*}^{\text {vir }} \frac{c_{\text {top }}\left(E_{\beta}\right)}{\hbar(\hbar-\psi)},
$$

which will be called $J$-function of $X$ and $E \rightarrow X$ respectively. It is easy to see that

$$
\int_{X} J_{X}^{E}=\sum_{\beta} q^{\beta} \int_{\left[\bar{M}_{0,1}(X, \beta)\right]^{\mathrm{vir}}} \frac{c_{\mathrm{top}}\left(E_{\beta}\right)}{\hbar(\hbar-\psi)} \mathrm{ev}^{*} e^{\frac{1}{\hbar}\left(t_{0}+\sum_{i} p_{i} t_{i}\right)}
$$

For a line bundle $L:=i^{*}\left(\mathcal{O}_{P}(l)\right)$ on $X$ define

$$
H_{\beta}^{L}:=\prod_{k=0}^{\left\langle c_{1}(L), \beta\right\rangle}\left(c_{1}(L)+k \hbar\right)
$$

for $L$ convex and

$$
H_{\beta}^{L}:=\prod_{k=\left\langle c_{1}(L), \beta\right\rangle+1}^{-1}\left(c_{1}(L)+k \hbar\right)
$$

for $L$ concave. Here a vector bundle $E \rightarrow X$ is called convex if for any stable map $f: C \rightarrow X, H^{1}\left(C, \pi_{*} f^{*}(E)\right)=0 . E$ is concave if $H^{0}\left(C, \pi_{*} f^{*}(E)\right)=0$ for any $f$. Introduce another generating 0 of Gromov-Witten invariants on $X$ (modified by $H^{L}$ 's)

$$
I_{X}^{E}(q, \hbar):=e^{\frac{1}{\hbar}\left(t_{0}+\sum_{i} p_{i} t_{i}\right)} \sum_{\beta} q^{\beta} J_{X}(\beta) \prod_{j} H_{\beta}^{L_{j}}
$$

Theorem 1. Let $X$ be a smooth projective variety embedded in $P=\prod \mathbb{P}^{r_{i}}$ (see Notations.) and $E=\oplus_{j} L_{j} \rightarrow X$ be the sum of line bundles $L_{j}$ which are the pull-backs of convex and concave line bundles on $P$ such that

$$
c_{1}\left(T_{X}\right)-\sum_{L_{j} \text { convex }} c_{1}\left(L_{j}\right)+\sum_{L_{j} \text { concave }} c_{1}\left(L_{j}\right)
$$


are non-negative. Then

$$
\int_{X} J_{X}^{E}(q, \hbar) \simeq \int_{X} I_{X}^{E}(q, \hbar)
$$

where $\simeq$ means equivalence up to a mirror transformation, which is a special kind of change of variables described in $\S 4.3$.

In fact, there are many situations when the above mirror transformations are unnecessary. The following theorem contains the main examples.

Theorem 2. $\int_{X} J_{X}^{E}=\int_{X} I_{X}^{E}$ if

1. $E$ is concave and $\operatorname{rank}(E) \geq 2$.

2. E is convex and $c_{1}\left(T_{X}\right)-\sum_{L_{j} \text { convex }} c_{1}\left(L_{j}\right)$ is Fano of index $\geq 2$.

3. Direct sum of the previous two cases.

In the case when $E:=\left(\oplus_{j} L_{j}\right)$, all $L_{j}$ are convex, Theorem 11 has the following interpretation.

Corollary 1. Let $i_{Y}: Y \hookrightarrow X$ be the smooth zero locus of a section of $E$ (i.e. a complete intersection in $X$ ).

1.

$$
\int_{X}\left(i_{Y}\right)_{*} J_{Y}=\int_{X} J_{X}^{E}
$$

In particular, Theorem 1 and Theorem 2 relate the Gromov-Witten invariants of $Y$ to Gromov-Witten invariants of $X$.

2. Suppose that $H^{*}(X)$ is generated by divisor classes, and $\operatorname{rk}(E)=1$, i.e. $Y$ is a hypersurface. Then all n-point gravitational Gromov-Witten invariants of $i_{Y}^{*} H^{*}(X)$ can be reconstructed from one-point gravitational Gromov-Witten invariants of $X$.

Proof. The part 1 is nothing but the statement that for any $\omega \in H^{*}(X)$

$$
\left\langle\left[\bar{M}_{0,1}(Y, \beta)\right]^{\mathrm{vir}}, i_{Y}^{*}(\omega)\right\rangle=\left\langle\left[\bar{M}_{0,1}(X, \beta)\right]^{\mathrm{vir}} \cap c_{\mathrm{top}}\left(E_{\beta}\right), \omega\right\rangle
$$

which can be found in e.g. [6] and references therein. See also $\S$ 1.2. The second part is a corollary of the first part plus a reconstruction theorem proved in [17], which states that one can reconstructs $n$-point descendants provided that $H^{*}(X)$ is generated by divisor classes and one-point descendants are known.

Remark 1. (Local mirror conjecture) When $E$ is concave, there is also an interpretation of the Gromov-Witten invariants of $E \rightarrow X$ : the invariants of the total space of vector bundle $E \rightarrow X$. For example $\mathcal{O}(-1) \oplus \mathcal{O}(-1) \rightarrow \mathbb{P}^{1}$, is the "tubular neighborhood" of $\mathbb{P}^{1}$ embedded in a Calabi-Yau threefold with normal bundle $\mathcal{O}_{\mathbb{P}^{1}}(-1) \oplus \mathcal{O}_{\mathbb{P}^{1}}(-1)$.

Remark 2 . Whenever $X$ carries a group action by $G$, one could carry out the whole work to $H_{G}^{*}(X)$ (instead of $H^{*}(X)$ ) without any change. In this case, only localization of $\mathbb{C}^{*}$-action on graph space is needed.

Combining the quantum differential equation [1] and the above theorems, one can easily see the following interesting phenomenon. When $X$ is a toric variety this is a corollary of Givental's quantum Serre duality theorem [12]. 
Corollary 2. Let $E=\oplus_{j=1}^{\mathrm{rk}(E)} L_{j}$ be a concave bundle with $\operatorname{rk}(E) \geq 2$. Then

$$
\int_{X} c_{\mathrm{top}}(E) e^{\frac{1}{\hbar}\left(t_{0}+p t\right)} J_{X}^{E}(q, \hbar) \sim \int_{X} \frac{1}{c_{\mathrm{top}}\left(E^{\vee}\right)} e^{\frac{1}{\hbar}\left(t_{0}+p t\right)} J_{X}^{E^{\vee}}(q, \hbar),
$$

where $E^{\vee}$ is the dual vector bundle and $\sim$ means equivalence up to mirror transformations (and a factor of power series in $q$ ).

An interesting consequence of (5) is that one can prove the mirror conjecture of convex bundles by concave bundles. For example the proof of mirror conjecture in the case of quintic three-fold can be carried outf by using $\mathcal{O}(-5)$ instead of $\mathcal{O}(5)$ on $\mathbb{P}^{4}$. See [12] $\S 5$ for an example. Here of course, this comes as a cyclic argument as we have used the proof of mirror conjecture in convex case to deduce this result. It is therefore desirable to have a direct proof of (5) and refine the statement. We plan to elaborate on this in a future paper (jointly with A. Bertram).

1.2. Relation to quantum cohomology. Lefschetz hyperplane theorem (LHT) asserts that a projective smooth variety $X$ contains essential (co-)homological information of its hyperplane section $Y$. The quantum Lefschetz hyperplane theorem (QLHT) verifies this assertion in quantum cohomology. It was proposed by A. Givental and formulated in the present form by B. Kim [15].

More precisely, our theorems state that the $J$-function of the complete intersection $Y$ (of classes $i_{Y}^{*} H^{*}(X)$ ) can be obtained from the $J$-function of $X$ by multiplying suitable cohomology classes and possibly a well-defined change of variables. The central roles of $J$-function in quantum cohomology theory is explained by the theory of quantum differential equation developed by Dijkgraaf and Givental [11]. It says, first of all, that $J$-function is a flat section of the Dubrovin connection on the A-model side, parallel to the Picard-Fuchs equation on the B-model side. This is related to the mirror symmetry discussed in the next subsection. Secondly, it gives a nice way to obtain the essential information of small quantum $\operatorname{ring} Q H^{*}(X)$ from $J$-function (quantum $\mathcal{D}$-module). For example, one can easily obtain the relations in quantum cohomology from $J$-function. Moreover, a result in 17 states that it is possible to reconstruct n-point descendants from one-point descendants when $H^{*}(X)$ is generated by divisor classes. Therefore our theorem even implicitly relates their big gravitational quantum cohomology algebras under this condition.

Many important special cases of QLHT, including the celebrated quintic three-

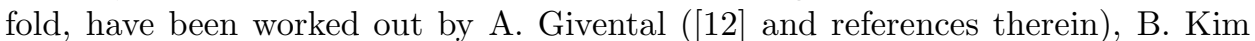
15 and Liu-Lian-Yau [19, 20, 21]. In the case of quintic three-fold, the ambient space $X=\mathbb{P}^{4}$ and $E=\mathcal{O}(5)$. Since it is very easy to compute the Gromov-Witten invariants of $\mathbb{P}^{4}$, QLHT is therefore the central part of the proof of mirror conjecture which will be discussed in the next subsection.

1.3. Relation to mirror conjecture. In a seminal paper [5] Candelas, de la Ossa, Green and Parkes applied the mirror symmetry to the quintic three-fold and derived, in a string-theoretic way, the celebrated formula which predicts the number $n_{d}$ of rational curves on the quintic three-fold of any degrees. This formula was then named mirror conjecture, or mirror identity to distinguish itself from more

\footnotetext{
${ }^{1}$ The restriction $\operatorname{rk}(E) \geq 2$ is shown unnecessary in the joint work with A. Bertram.

${ }^{2}$ Here we are not very precise. $(5)$ is valid only if $\operatorname{rk}(E) \geq 2$. However, we could use $E=$ $\mathcal{O}(-5) \oplus \mathcal{O}(-1)$ on $\mathbb{P}^{5}$ instead of $\mathcal{O}(-5)$ on $\mathbb{P}^{4}$. The reason is that quintic three-fold can be described as complete intersections in $\mathbb{P}^{5}$ of the bundle $\mathcal{O}(1) \oplus \mathcal{O}(5)$.
} 
fundamental physical principle of mirror symmetry. This conjecture basically says that a generating function of $n_{d}$ is equivalent to a hypergeometric series up to a mirror transformation. Their result soon stimulated a lot of mathematical work in enumerative geometry. Among different groups working on the proof of their prediction, there have been notably two different approaches. One approach is trying to mathematically justify the string-theoretic mirror symmetry and therefore obtain mirror conjecture as a corollary. The other is to attack the enumerative consequence directly by developing new mathematics inspired from physics. GromovWitten theory is partly inspired by this second approach which we will give a brief discussion?.

The first major progress came from M. Kontsevich [16]. As is well known in algebraic geometry, an enumerative problem can usually be formulated as an intersection-theoretic one on suitable moduli spaces. Kontsevich introduced the moduli space of stable maps and formulated the mirror conjecture as follows. Let $\mathcal{O}(5)_{d}^{\prime}:=\pi_{*} \operatorname{ev}^{*}(\mathcal{O}(5))$ be the vector bundle on $\bar{M}_{0,0}\left(\mathbb{P}^{4}, d\right)$, then the enumerative problem was equivalent to computing the integral

$$
N_{d}:=\int_{\bar{M}_{0,0}\left(\mathbb{P}^{4}, d\right)} c_{\mathrm{top}}\left(\mathcal{O}(5)_{d}^{\prime}\right),
$$

and $N_{d}$ can be related to $n_{d}$ by Aspinwall-Morrison Formula. By using torus action on $\mathbb{P}^{4}$ and fixed point localization method, he was able to reduce the integral (6) to summation of trees, but failed to complete the complicated combinatorial problem. Another (conceptual) drawback of this approach was that it did not explain the presence of hypergeometric series.

Then came A. Givental's proof followed by other approaches and generalizations by Lian-Liu-Yau and Bertram. The new innovations include, among other things, the introduction of equivariant quantum cohomology and graph space. The quantum cohomology of Calabi-Yau manifold $X$ is not semisimple, which makes the structure of quantum ring, like associativity relation, not very useful in computing $n_{d}$. By introducing equivariant quantum cohomology one produces a family of Frobenius structure over $H_{G}^{*}(p t)$ whose generic fibre carries semisimple Frobenius structure while the special fibre $H^{*}(Y)$ does not. Therefore $Q H^{*}(Y)$ for CalabiYau manifold $Y$ may be considered as a limiting case of semisimple Frobenius manifold. This explains, in one way, why the structure of quantum cohomology of Calabi-Yau manifolds play a role in enumerative problem. On the other hand, to properly explain the presence of hypergeometric series, the graph space $\bar{G}_{0,0}\left(\mathbb{P}^{r}, d\right)$ was introduced and was shown to have a natural birational morphism $u$ to the toric compactification space, or linear sigma model, $\mathbb{P}_{d}^{r}:=\mathbb{P}^{(r+1) d+r}$ (see $\S 2.1$ for details). It was earlier found by E. Witten 23] and Givental, etc. (from different approaches) that some suitable correlators on $\mathbb{P}_{d}^{r}$ actually produce the desired hypergeometric series. However, neither $\bar{G}_{0,0}\left(\mathbb{P}^{r}, d\right)$ nor $\mathbb{P}_{d}^{r}$ is the right space to perform the integral (6). The way to resolve this issue was to identify $\bar{M}_{0,1}\left(\mathbb{P}^{r}, d\right)$ as a fixed point component of $\mathbb{C}^{*}$-action on $\bar{G}_{0,0}\left(\mathbb{P}^{r}, d\right)$. One then uses the birational morphism $u: \bar{G}_{0,0}\left(\mathbb{P}^{r}, d\right) \rightarrow \mathbb{P}_{d}^{r}$ to pass the above correlators from $\mathbb{P}_{d}^{r}$ to $\bar{G}_{0,0}\left(\mathbb{P}^{r}, d\right)$ and then pass to $\bar{M}_{0,1}\left(\mathbb{P}^{r}, d\right)$. In the quintic three-fold case, $r=4$ and

\footnotetext{
${ }^{3}$ The following is not meant to be a precise historical account.
} 
the correlator obtained from this procedure is

$$
\begin{aligned}
J_{\mathbb{P}^{4}}^{\mathcal{O}(5)}(d) & :=\mathrm{ev}_{*} \frac{c_{\mathrm{top}}\left(\mathcal{O}(5)_{d}\right)}{\hbar(\hbar-\psi)} \\
& =\hbar^{-2} \mathrm{ev}_{*} c_{\mathrm{top}}\left(\mathcal{O}(5)_{d}\right)+\hbar^{-3} \mathrm{ev}_{*}\left(c_{\mathrm{top}}\left(\mathcal{O}(5)_{d}\right) \psi\right)+\cdots
\end{aligned}
$$

The $\hbar^{-2}$ term in Laurent series expansion, when integrated over $\mathbb{P}^{4}$, will be (see 19)

$$
\int_{\mathbb{P}^{4}} \mathrm{ev}_{*} c_{\mathrm{top}}\left(\mathcal{O}(5)_{d}\right)=\int_{\bar{M}_{0,1}\left(\mathbb{P}^{4}, d\right)} c_{\mathrm{top}}\left(\mathcal{O}(5)_{d}\right)=\int_{\bar{M}_{0,0}\left(\mathbb{P}^{4}, d\right)} c_{\mathrm{top}}\left(\mathcal{O}(5)_{d}^{\prime}\right)
$$

which is exactly (6).

There are now four approaches to mirror conjecture (known to us) by Givental, Lian-Liu-Yau, Bertram, and Gathmann. The interested reader can find valuable information in 12, 15, 21, 3, 9] and references therein.

Remark 3. Compare (7) with (1), one sees that our results can be interpreted as a generalization of mirror conjecture.

Acknowledgements. My special thanks go to Aaron Bertram. The current proof is based on his work 3 and I benefit a lot from our collaboration. I am also thankful to Bumsig Kim 7 , Rahul Pandharipande for numerous useful discussions.

Update. Gathmann has recently posted his proof of mirror theorem [10], where he proved the mirror theorem in the case $E$ is a convex line bundle (i.e. when $Y$ is a very ample hypersurface). The relation between his approach and ours is, roughly, the following. While we tried to sweep the classes $e_{\nu}$ in Theorem 5 "under the carpet" by dimensional constraints, he explicitly studies these relative classes and found a nice formula to relate these classes to ordinary Gromov-Witten classes.

\section{Graph space in Gromov-Witten theory}

The Picard number of $X$ is assumed to be one throughout the rest of the paper, to avoid complicated notations. The generalization to the arbitrary Picard number is usually a matter of bookkeeping and is left to the reader.

2.1. Graph space and one-point invariants. The $n$-pointed graph space of $X$ of degree $\beta$ is defined to be $\bar{G}_{0, n}(X, \beta):=\bar{M}_{0, n}\left(X \times \mathbb{P}^{1},(\beta, 1)\right)$, where the degree $(\beta, 1)$ is the element in $H_{2}(X) \oplus H_{2}\left(\mathbb{P}^{1}\right)$. It is a compactification of the space of maps from parameterized $\mathbb{P}^{1}$ to $X$. This space $\bar{G}_{0, n}(X, \beta)$ carries a natural $\mathbb{C}^{*}$ action induced from the action on $\mathbb{P}^{1}$.

When $X=\mathbb{P}^{r}$ there is another (toric) compactification, $\mathbb{P}_{d}^{r}$ (linear sigma model), of the space of parameterized map of degree $d$. It is constructed in the following way. Consider the projective space of $(r+1)$-tuple of the degree $d$ (symmetric) binary forms of $\left(z_{0}: z_{1}\right)$. When there is no common factors of positive degrees among these $(r+1)$-tuples, it represents a morphism from $\mathbb{P}^{1} \rightarrow \mathbb{P}^{r}$. We may compactify it by simply allowing the common factors and taking quotient by $\mathbb{C}^{*}$-action. It is easy to see that this space is equal to $\mathbb{P}^{(r+1)(d+1)-1}$.

By construction $\bar{G}_{0,0}\left(\mathbb{P}^{r}, d\right)$ is birationally isomorphic to $\mathbb{P}_{d}^{r}$. In fact,

\footnotetext{
${ }^{4}$ B. Kim informed us that he had previously obtained a special case of Theorem 1 case
} 
Theorem 3. (Givental's Main Lemma [1]) There exists a natural birational $\mathbb{C}^{*}$ equivariant morphism $u: \bar{G}_{0,0}\left(\mathbb{P}^{r}, d\right) \rightarrow \mathbb{P}_{d}^{r}$.

This morphism $u$ can be described as follows. Consider a stable degree $(d, 1)$ map $f: C \rightarrow \mathbb{P}^{1} \times \mathbb{P}^{n}$. There exists a unique irreducible component $C_{0} \in C$ (called parameterized component) such that $\left.f\right|_{C_{0}}$ has degree $\left(d_{0}, 1\right)$ where $d_{0} \leq d$. The image $f\left(C_{0}\right)$ is the graph of a map $\mathbb{P}^{1} \rightarrow \mathbb{P}^{r}$ of degree $d_{0}$. The map is given by the binary forms $\left(p_{0}: \cdots: p_{r}\right)$ of degree $d_{0}$ with no common factors and determines the forms uniquely up to a non-zero constant factor. The curve $\overline{C \backslash C_{0}}$ has $s$ connected unparameterized components which are mapped to $\mathbb{P}^{1} \times \mathbb{P}^{r}$ with degrees $\left(d_{1}, 0\right), \ldots,\left(d_{s}, 0\right), d_{1}+\ldots+d_{s}=d-d_{0}$, and the image of $i$-th component is contained in the slice $\left(a_{i}: b_{i}\right) \times \mathbb{P}^{n}$. We put $u(C, f)=\prod_{i=1}^{r}\left(a_{i} z_{0}-b_{i} z_{1}\right)^{d_{i}}\left(p_{0}: \cdots: p_{r}\right)$. For a detailed proof in algebro-geometric terms see [19] and [4].

Of course we can also find simple birational models for $\bar{G}_{0, s}\left(\mathbb{P}^{r}, d\right)$. A particular useful one is $\left(\mathbb{P}^{1}\right)^{s} \times \mathbb{P}_{d}^{r}$. There is also a morphism $u_{s}: \bar{G}_{0, s}\left(\mathbb{P}^{r}, d\right) \rightarrow\left(\mathbb{P}^{1}\right)^{s} \times \mathbb{P}_{d}^{r}$. The first factor is defined by the composition

$$
\bar{G}_{0, s}\left(\mathbb{P}^{r}, d\right) \stackrel{\prod \mathrm{ev}_{i}}{\longrightarrow}\left(X \times \mathbb{P}^{1}\right)^{s} \stackrel{\prod p_{2}}{\longrightarrow}\left(\mathbb{P}^{1}\right)^{s}
$$

and the second factor is the composition of the forgetful morphism $\bar{G}_{0, s}\left(\mathbb{P}^{r}, d\right) \rightarrow$ $\bar{G}_{0,0}\left(\mathbb{P}^{r}, d\right)$ and $u: \bar{G}_{0,0}\left(\mathbb{P}^{r}, d\right) \rightarrow \mathbb{P}_{d}^{r}$ defined in Theorem 3 .

From the above description, it is clear that two spaces $\bar{G}_{0,0}\left(\mathbb{P}^{r}, d\right)$ and $\mathbb{P}_{d}^{r}$ differ on certain boundary strata. The comb type strata, denoted $D_{\mu}$ where $\mu:=$ $\left(d_{0}, d_{1}, \cdots, d_{s}\right), d_{0}+d_{1}+\cdots d_{s}=d$, play an important role in our discussion. The (domain) curve of a generic element in $D_{\mu}$ has one parameterized component and $s$ nodes, and $\left(d_{0}, 1\right) \in H_{2}\left(\mathbb{P}^{r} \times \mathbb{P}^{1}\right)$ is the degree of the parameterized component. These strata have substrata, called hairy comb type strata, which are obtained by further degenerating the unparameterized components. Note that different permutations of $d_{1}, \cdots, d_{s}$ represent the same $\mu$. For example $\left(d_{0}, d^{\prime}, d^{\prime \prime}\right)=\left(d_{0}, d^{\prime \prime}, d^{\prime}\right)$. For such a stratum $D_{\mu}$ there is a finite birational morphism

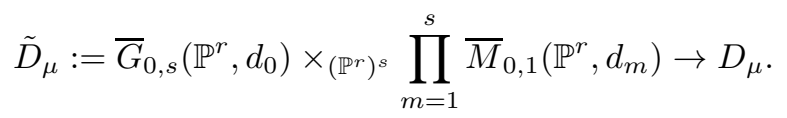

To simplify our notation, we will denote $\tilde{D}_{\mu}$ also by $D_{\mu}$ henceforth. There are also useful morphisms from $D_{\mu}$ to simple spaces:

$$
u_{\mu}: D_{\mu}=\bar{G}_{0, s}\left(\mathbb{P}^{r}, d_{0}\right) \times{ }_{\left(\mathbb{P}^{r}\right)^{s}} \prod_{m=1}^{s} \bar{M}_{0,1}\left(\mathbb{P}^{r}, d_{m}\right) \rightarrow\left(\mathbb{P}^{1}\right)^{s} \times \mathbb{P}_{d_{0}}^{r}
$$

defined by the composition

$$
D_{\mu} \stackrel{p_{0}}{\rightarrow} \bar{G}_{0, s}\left(\mathbb{P}^{r}, d_{0}\right) \stackrel{u_{\Im}}{\rightarrow}\left(\mathbb{P}^{1}\right)^{s} \times \mathbb{P}_{d_{0}}^{r} .
$$

Let $X \stackrel{i}{\hookrightarrow} \mathbb{P}^{r}$ be an embedding described earlier. For the notational convenience, we define the divisors $D_{\nu}:=i_{G}^{*} D_{\mu}$ of $\bar{G}_{0,0}(X, \beta)$ (indexed by $\nu:=\left(\beta_{0}, \beta_{1}, \cdots, \beta_{s}\right)$ ) 
for future reference. The relation is described in the following commutative diagram:

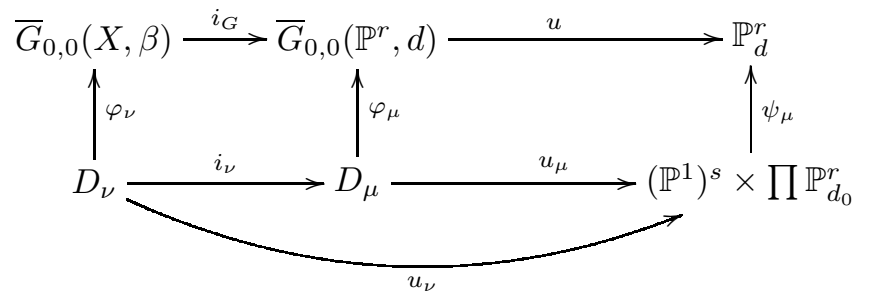

The reason to introduce the graph space in Gromov-Witten theory is to obtain one-point descendant invariants. The graph space $\bar{G}_{0,0}(X, \beta)$ carries a $\mathbb{C}^{*}$ action induced from the $\mathbb{C}^{*}$ action on $\mathbb{P}^{1}$, and $\bar{M}_{0,1}(X, \beta)$ is a fixed point component. It is summarized in the following commutative diagram:

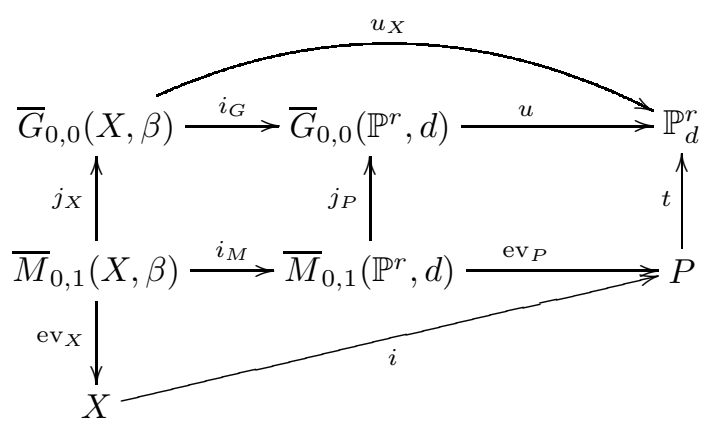

where the left upper square is a commutative diagram such that $\bar{M}_{0,1}(X, \beta) \hookrightarrow$ $\bar{G}_{0,0}(X, \beta)$ and $\bar{M}_{0,1}(P, d) \hookrightarrow \bar{G}_{0,0}(P, d)$ are fixed point components of $\mathbb{C}^{*}$-action on the graph spaces and $\bar{M}_{0,1}(X, \beta)$ is the only fixed point component mapping to $\bar{M}_{0,1}\left(\mathbb{P}^{r}, d\right)$ by $i_{M}$. The same can be said about the right square: $\mathbb{P}^{r} \hookrightarrow \mathbb{P}_{d}^{r}$ is a fixed point component such that $\bar{M}_{0,1}\left(\mathbb{P}^{r}, d\right)$ is the only fixed point component mapping to $\mathbb{P}^{r}$.

Remark 4 . The above setting actually works for product of projective spaces $P:=$ $\prod \mathbb{P}^{r_{i}}$. There are only small changes in this adjustment. First, all $d, \mu$ etc. should stand for multi-index. Second, the birational morphism in Theorem 3 should be replaced by

$$
u: \bar{G}_{0,0}(P, d) \rightarrow \prod_{i} \mathbb{P}_{d^{i}}^{r_{i}}
$$

2.2. Virtual localization on graph space. First recall the Graber-Pandharipande virtual localization formula [14]:

Theorem 4. Let $X$ be an algebraic scheme with a $\mathbb{C}^{*}$ action and $\mathbb{C}^{*}$-equivariant perfect obstruction theory. Then the virtual localization formula holds:

$$
[X]^{\mathrm{vir}}=j_{*} \sum \frac{\left[X_{j}\right]^{\mathrm{vir}}}{e\left(N_{X_{j} \mid X}^{\mathrm{vir}}\right)}
$$

in $A_{*}^{\mathbb{C}^{*}}(X) \otimes \mathbb{Q}\left[\lambda, \frac{1}{\lambda}\right]$, where $\lambda$ is the generator of the $A_{*}^{\mathbb{C}^{*}}(p t)$. 
An immediate consequence of this theorem is the correspondence of residues ([21] Lemma 2.1 and [3]):

Corollary 3. Suppose that $f: X_{1} \rightarrow X_{2}$ is a $\mathbb{C}^{*}$-equivariant map of two algebraic schemes and $j_{1}: F_{1} \rightarrow X_{1}$ and $j_{2}: F_{2} \rightarrow X_{2}$ are two fixed point components of $X_{1}$ and $X_{2}$ respectively, such that $F_{1}$ is the only fixed point component mapping into $F_{2}$ as in the following commutative diagram:

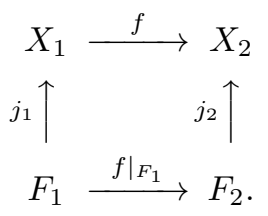

Then

$$
\left.f\right|_{F_{1 *}}\left(\frac{j_{1}^{*}(\omega) \cap\left[F_{1}\right]^{\mathrm{vir}}}{e\left(N_{F_{1} \mid X_{1}}^{\mathrm{vir}}\right)}\right)=\frac{j_{2}^{!} f_{*}\left(\omega \cap\left[X_{1}\right]^{\mathrm{vir}}\right)}{e\left(N_{F_{2} \mid X_{2}}^{\mathrm{vir}}\right)}
$$

for any $\omega \in H_{\mathbb{C}^{*}}^{*}\left(X_{1}\right)$.

Apply this result to our case: Let $X_{1}=\bar{G}_{0,0}(X, \beta), X_{2}=\bar{G}_{0,0}(P, d), F_{1}=$ $\bar{M}_{0,1}(X, \beta), F_{2}=\bar{M}_{0,1}(P, d)$ and $\omega=c_{\text {top }}\left(E_{\beta}^{G}\right)$, as displayed in the upper left square of (11). Since $j_{X}^{*} c_{\text {top }}\left(E_{\beta}^{G}\right)=c_{\text {top }}\left(E_{\beta}\right)$, one has

$$
\begin{aligned}
i_{M *}^{\mathrm{vir}}\left(\frac{c_{\mathrm{top}}\left(E_{\beta}\right)}{\hbar(\hbar-\psi)}\right) & =\operatorname{PD} i_{M *}\left(\frac{c_{\mathrm{top}}\left(E_{\beta}\right)}{\hbar(\hbar-\psi)} \cap\left[\bar{M}_{0,1}(X, \beta)\right]^{\mathrm{vir}}\right) \\
& =\frac{\operatorname{PD} j_{P}^{!} i_{G *}\left(c_{\mathrm{top}}\left(E_{\beta}^{G}\right) \cap\left[\bar{G}_{0,0}(X, \beta)\right]^{\mathrm{vir}}\right)}{\hbar(\hbar-\psi)} \\
& =\frac{j_{P}^{*} i_{G *}^{\mathrm{vir}} c_{\mathrm{top}}\left(E_{\beta}^{G}\right)}{\hbar(\hbar-\psi)}
\end{aligned}
$$

where $\hbar$ is the generator of $H_{\mathbb{C}^{*}}^{*}(p t)$ and $\psi$ is the first chern class of the tautological line bundle $\mathcal{L}_{1}$ on $\bar{M}_{0,1}(X, \beta)$ or $\bar{M}_{0,1}(P, d)$. The one small difference between (12) and $(13)$ is that $\bar{G}_{0,0}(P, d)$ and $\bar{M}_{0,1}(P, d)$ are orbifolds and Poincaré duality makes sense there.

Remark 5. The functorial properties of virtual fundamental classes used in this article can be found in [18] [2] [1].

\section{DeCOMposition OF the VIRTUAL FUndAMENTAL ClASSES}

Recall that there is a birational morphism $u_{X, 1}:=u_{1} \circ i_{G}$ (see (8)) from the universal curve $\bar{G}_{0,1}(X, \beta)$ of the graph space $\bar{G}_{0,0}(X, \beta)$ to $\mathbb{P}^{1} \times \mathbb{P}_{d}^{r}$. Given a line bundle $L:=i^{*} \mathcal{O}_{\mathbb{P}^{r}}(l)$ on $X$ one could produce two line bundles $\mathrm{ev}^{*} L$ and $u_{X, 1}^{*} \mathcal{O}_{\mathbb{P}^{1} \times \mathbb{P}_{d}^{r}}(d l, l)$ on $\bar{G}_{0,1}(X, \beta)$. These two line bundles are isomorphic on the open subset $U:=\bar{G}_{0,1}(X, \beta) \backslash \cup_{\nu=\left(\beta_{0}, \beta_{1}\right)} C_{\nu}$, where $C_{\nu}$ is the universal curve of the unparameterized component over $D_{\nu}$. The reason is that $C_{\nu}$ are exactly the exceptional divisors of $u_{1}$. More explicitly, there is a rational map $b: \mathbb{P}^{1} \times \mathbb{P}_{d}^{r} \rightarrow \mathbb{P}^{r}$ 
Y.-P. LEE

such that $b$ is well-defined on $u(U)$ in the following commutative diagram:

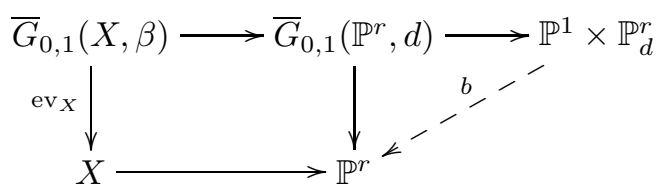

It is easy to see that $b$ is a morphism on $u(U)$, of degree $d$ in the first factor and linear in the second. Namely

$$
\left.b\right|_{u(U)} ^{*} \mathcal{O}_{\mathbb{P}^{r}}(l)=\mathcal{O}_{\mathbb{P}^{1} \times \mathbb{P}_{d}^{r}}(d l, l) .
$$

Therefore

$$
\operatorname{ev}_{X}^{*}(L)=u_{X, 1}^{*}\left(\mathcal{O}_{\mathbb{P}^{1} \times \mathbb{P}_{d}^{r}}(d l, l)\right) \otimes \mathcal{O}\left(\sum_{\nu=\left(\beta_{0}, \beta_{1}\right)} c_{\nu} C_{\nu}\right) .
$$

It is easy to see that $c_{\nu}$ is negative as $\left.\operatorname{ev}_{X}^{*}(L) \hookrightarrow \mathcal{O}_{\mathbb{P}^{1} \times \mathbb{P}_{d}^{r}}(d l, l)\right)$ by restricting a section of $\mathrm{ev}_{X}^{*}(L)$ to $U$ and then extending this section on $\mathbb{P}_{d}^{r}$ by Hartog's lemma. The actual coefficients $c_{\nu}$ can be determined by the following observation. The degree of $f^{*}(L)$ on the unparameterized component of the universal curve over the divisor $D_{\nu}$ (at a generic point) has degree $\left\langle c_{1}(L), \beta_{1}\right\rangle$. This leads to $c_{\nu}=$ $-\left\langle c_{1}(L), \beta_{1}\right\rangle$. The same argument applies to $n$-pointed graph space:

Lemma 1. On the universal curve $C$ over the graph space $\bar{G}_{0, n}(X, \beta)$

$$
\begin{aligned}
& \operatorname{ev}_{1, X}^{*}(L) \\
= & u_{X, n}^{*}\left(\mathcal{O}_{\mathbb{P}^{1} \times\left(\mathbb{P}^{1}\right)^{n} \times \mathbb{P}_{d}^{r}}(d l, 0, \cdots, 0, l)\right) \otimes \mathcal{O}\left(\sum_{\nu=\left(\beta_{0}, \beta_{1}\right)}-\left\langle c_{1}(L), \beta_{1}\right\rangle C_{\nu}\right),
\end{aligned}
$$

where $C_{\nu}$ is the universal curve of the unparameterized component over $D_{\nu}$.

Corollary 4. For L convex let

$$
\begin{gathered}
L_{\beta}^{G}:=R^{0} \pi_{n+1 *} \operatorname{ev}_{n+1}^{*}\left(L_{l}\right) \\
F_{\beta}^{G}:=u_{X}^{*}\left(H^{0}\left(\mathbb{P}^{1}, \mathcal{O}(d l)\right) \otimes \mathcal{O}_{\left(\mathbb{P}^{1}\right)^{n} \times \mathbb{P}_{d}^{r}}(0, \cdots, 0, l)\right) .
\end{gathered}
$$

One has equivariant maps of vector bundles on graph space $\bar{G}_{0, n}(X, \beta)$

$$
\sigma_{0}: L_{\beta}^{G} \rightarrow F_{\beta}^{G}
$$

For $L$ concave let

$$
\begin{gathered}
L_{\beta}^{G}:=R^{1} \pi_{*} e^{*}\left(L_{l}\left(-x_{1}-\cdots-x_{n}\right)\right) \\
F_{\beta}^{G}:=u_{X}^{*}\left(H^{1}\left(\mathbb{P}^{1}, \mathcal{O}(d l)\left(-\chi_{1}-\cdots-\chi_{n}\right)\right) \otimes \mathcal{O}_{\left(\mathbb{P}^{1}\right)^{n} \times \mathbb{P}_{d}^{r}}(0, l)\right) .
\end{gathered}
$$

One has

$$
\sigma_{1}: F_{\beta}^{G} \rightarrow L_{\beta}^{G}
$$

Here in $14 \mathrm{~b}) \chi_{m}:\left(\mathbb{P}^{1}\right)^{n} \otimes \mathbb{P}_{d}^{r} \rightarrow \mathbb{P}^{1}$ are the projections to the $m$-th factor of $\mathbb{P}^{1}$ (considered as "marked points"). In equation (14a), $x_{m}$ are the marked points on the universal curve.

\footnotetext{
${ }^{5}$ In fact, we will only need to know $c_{\nu}$ is negative.
} 
Proof. It is clear that the vector bundles in this lemma are the push-forwards of two line bundles on the universal curve $C$ considered in Lemma ti. When the coefficient $c_{\nu}$ is negative, one has the following inclusion

$$
\operatorname{ev}_{X}^{*}(L) \hookrightarrow u_{X, n}^{*} \mathcal{O}_{\mathbb{P}^{1} \times\left(\mathbb{P}^{1}\right)^{n} \times \mathbb{P}_{d}^{r}}(d l, 0, d) .
$$

$\sigma_{0}$ is then obtained by pushing-forward the above inclusion of line bundles to $\bar{G}_{0, n}(X, \beta)$.

$\sigma_{1}$ can be obtained in a similar way. When $L$ is concave, $-\left\langle c_{1}(L), \beta_{1}\right\rangle$ is positive so that the arrow of (15) is reversed.

The equation (1) indicates that the QLHT boils down to the study of the top chern class $c_{\text {top }}\left(E_{\beta}\right)$ on $\bar{M}_{0,1}(X, \beta)$, which is in turn the pull-back $j_{X}^{*}\left(c_{\text {top }}\left(E_{\beta}^{G}\right)\right)$ of top chern class from $\bar{G}_{0,0}(X, \beta)$. From the above discussion

$$
c_{\text {top }}\left(E_{\beta}^{G}\right)=u_{X}^{*} c_{\text {top }}\left(F_{\beta}^{G}\right)+\text { boundary terms, }
$$

where the boundary terms are supported on the comb type strata $D_{\nu}$. Notice that the "main term" $c_{\text {top }}\left(F_{\beta}^{G}\right)$ is the (equivariant) top chern class of direct sum of line bundles on the projective space and can therefore be easily computed. In fact, we will see in the next section that this part gives rise to the factors $H_{\beta}^{E}$ in (3). It remains to have a close look of the boundary terms. A key observation of [3] is that boundary contributions can be explicitly computed using MacPherson's graph construction for vector bundle morphisms.

Proposition 1. When $X=\mathbb{P}^{r}$ and $E=\mathcal{O}_{\mathbb{P}^{r}}(l)$ a line bundle,

$$
c_{\mathrm{top}}\left(\mathcal{O}_{\mathbb{P}^{r}}(l)_{d}^{G}\right)=u^{*} \prod_{k=0}^{d l}(l H+k \hbar)+\sum_{\mu} \frac{1}{s !} \varphi_{\mu_{*}}\left(e_{\mu} \cup u_{\mu}^{*} \prod_{k=0}^{d_{0} l}(l H+k \hbar)\right),
$$

for $l$ positive and

$c_{\text {top }}\left(\mathcal{O}_{\mathbb{P}^{r}}(l)_{d}^{G}\right)=u^{*} \prod_{k=d l+1}^{-1}(l H+k \hbar)+\sum_{\mu} \frac{1}{s !} \varphi_{\mu_{*}}\left(e_{\mu} \cup u_{\mu}^{*} \xi_{1} \cdots \xi_{s} \prod_{k=d_{0} l+1}^{-1}(l H+k \hbar)\right)$,

for $l$ negative. Here $s$ is the number of unparameterized components for a generic curve over $D_{\nu}, H$ is the hyperplane class of $\mathbb{P}_{d}^{r}$ and $\xi_{m}$ is the point class of $m$-th $\mathbb{P}^{1}$ in $\left(\mathbb{P}^{1}\right)^{s} \times \mathbb{P}_{d}^{r}$. The equivariant class $e_{\mu}$ is defined in (23).

Theorem 5. Let $E=\oplus_{j} L_{j}, L_{j}=i^{*}\left(\mathcal{O}_{\mathbb{P}^{r}}\left(l_{j}\right)\right)$. The virtual fundamental class $c_{\mathrm{top}}\left(E_{\beta}^{G}\right) \cap\left[\bar{G}_{0,0}(X, \beta)\right]^{\mathrm{vir}}$ decomposes as follows:

For convex bundle $E$

$$
\begin{aligned}
& c_{\mathrm{top}}\left(E_{\beta}^{G}\right) \cap\left[\bar{G}_{0,0}(X, \beta)\right]^{\mathrm{vir}} \\
= & u_{X}^{*} \prod_{j} \prod_{k_{j}=0}^{\left\langle c_{1}\left(L_{j}\right), \beta\right\rangle}\left(l_{j} H+k_{j} \hbar\right) \cap\left[\bar{G}_{0,0}(X, \beta)\right]^{\mathrm{vir}} \\
+ & \sum_{\nu} \frac{1}{s !} \varphi_{\nu_{*}}\left(\left(e_{\nu} \cup u_{\nu}^{*} \prod_{j} \prod_{k_{j}=0}^{\left\langle c_{1}\left(L_{j}\right), \beta_{0}\right\rangle}\left(l_{j} H+k_{j} \hbar\right)\right) \cap\left[D_{\nu}\right]^{\mathrm{vir}}\right) .
\end{aligned}
$$


For concave $E$

$$
\begin{aligned}
& c_{\text {top }}\left(E_{\beta}^{G}\right) \cap\left[\bar{G}_{0,0}(X, \beta)\right]^{\mathrm{vir}} \\
= & u_{X}^{*} \prod_{j} \prod_{k_{j}=\left\langle c_{1}\left(L_{j}\right), \beta\right\rangle+1}^{-1}\left(l_{j} H+k_{j} \hbar\right) \cap\left[\bar{G}_{0,0}(X, \beta)\right]^{\mathrm{vir}} \\
+ & \sum_{\nu} \frac{1}{s !} \varphi_{\nu_{*}}\left(\left(e_{\nu} \cup u_{\nu}^{*} \prod_{j} \prod_{k_{j}=\left\langle c_{1}\left(L_{j}\right), \beta_{0}\right\rangle+1}^{-1} \xi_{1} \cdots \xi_{s}\left(l_{j} H+k_{j} \hbar\right)\right) \cap\left[D_{\nu}\right]^{\mathrm{vir}}\right) .
\end{aligned}
$$

In the case $\operatorname{rk}(E)=1, e_{\nu}:=i_{G}^{*} e_{\mu}$. In general, it is defined inductively.

Proof. It is easy to see that the theorem follows from the above proposition by the formula $c_{\text {top }}\left(E_{\beta}^{G}\right)=\prod_{j} c_{\text {top }}\left(\left(L_{j}\right)_{\beta}^{G}\right)$ and the excess intersection theory. Note that $c_{\mathrm{top}}\left(\left(L_{j}\right)_{\beta}^{G}\right)=i_{G}^{*} c_{\mathrm{top}}\left(\mathcal{O}_{\mathbb{P}^{r}}\left(l_{j}\right)_{d}^{G}\right)$.

Proof. (of Proposition [1)

I. (Convex case) The convex case of the proposition is Lemma 4.4 of [3]. For the convenience of the reader and future references, we reproduce Bertram's proof.

As remarked above, it is clear from Lemma 1 that the equivariant virtual class has its "main" contribution from

$$
c_{\mathrm{top}}\left(u^{*} \mathcal{O}_{\mathbb{P}^{1} \times \mathbb{P}_{d}^{r}}(l d, l)\right)=\left(u^{*} \prod_{k=0}^{l d}(l H+k \hbar)\right) .
$$

It is also clear that other contributions to $c_{\text {top }}\left(\mathcal{O}_{\mathbb{P}^{r}}(l)_{d}^{G}\right)$ come from the boundary strata $\mu$ (and possibly its substrata). It remains to study the boundary terms. This can be done by MacPherson's graph construction of vector bundle morphisms.

Let $\mathcal{E}_{d}:=\mathcal{O}_{\mathbb{P}^{r}}(l)_{d}^{G}$ and $\mathcal{F}_{d}:=H^{0}\left(\mathbb{P}^{1}, \mathcal{O}(d l)\right) \otimes u^{*} \mathcal{O}_{\mathbb{P}_{d}^{r}}(l)$ be vector bundles on $\bar{G}_{0,0}\left(\mathbb{P}^{r}, d\right)$. By Corollary 1 there is a homomorphism $\sigma_{0}: \mathcal{E}_{d} \rightarrow \mathcal{F}_{d}$ of vector bundles of the same rank $d l+1$. Let $G:=\operatorname{Grass}_{d l+1}\left(\mathcal{E}_{d} \oplus \mathcal{F}_{d}\right)$ be the Grassmann bundle over $\bar{G}_{0,0}\left(\mathbb{P}^{r}, d\right)$, with universal bundle $\zeta \rightarrow G$ of rank $d l+1$. There is a canonical embedding

$$
\Phi: \bar{G}_{0,0}\left(\mathbb{P}^{r}, d\right) \times \mathbb{A}^{1} \rightarrow G \times \mathbb{P}^{1}
$$

taking $(z, \lambda)$ to (graph of $\lambda \sigma_{0}(z),(1: \lambda)$ ). Let $W$ be the closure of the image of $\Phi$ and let

$$
W_{\infty}=i_{\infty}^{*}[W]=\sum m_{\delta}\left[V_{\delta}\right]
$$

be a cycle in $G$ of dimension equal to $\operatorname{dim}\left(\bar{G}_{0,0}\left(\mathbb{P}^{r}, d\right)\right)$, where $m_{\delta}$ is the multiplicity of $V_{\delta}$. Let

$$
\eta_{\delta}: V_{\delta} \subset G \rightarrow Z_{\delta} \subset \bar{G}_{0,0}\left(\mathbb{P}^{r}, d\right)
$$

be the map induced by projection, and $Z_{\delta}$ be the image of $\eta_{\delta}$. The philosophy of the graph construction is that different components $Z_{\delta}$ are responsible for different types of degeneration of $\sigma_{0}$. Since $\sigma_{0}$ is generically of full rank, there is one component $V_{0} \sim Z_{0} \cong \bar{G}_{0,0}\left(\mathbb{P}^{r}, d\right)$ in $W_{\infty}$ with multiplicity one such that $Z_{0}$ embeds in $G$ via the fibre of $\mathcal{F}_{d}$. Other $V_{\delta}$ map to proper sub-varieties $Z_{\delta}$ of $\bar{G}_{0,0}\left(\mathbb{P}^{r}, d\right)$. It 
follows that (河, Example 18.1.6)

$$
c_{\mathrm{top}}\left(\mathcal{E}_{d}\right)=u^{*} \prod_{k=0}^{d l+1}(l H+k \hbar)+\sum_{\delta} m_{\delta} \eta_{\delta_{*}} c_{\mathrm{top}}(\zeta) .
$$

To calculate the contributions from $V_{\delta}$, further study on the behavior of $\sigma_{0}$ on boundary strata $D_{\mu}$ is needed. Let $f: C \rightarrow \mathbb{P}^{r}$ be a generic stable map in strata $\mu=\left(d_{0}, \cdots, d_{r}\right) .\left.\sigma_{0}\right|_{D_{\mu}}$ can be described fibrewisely (at a generic point) as:

$$
H^{0}\left(C, f^{*} \mathcal{O}_{\mathbb{P}^{r}}(l)\right) \rightarrow H^{0}\left(C_{0},\left.f^{*} \mathcal{O}_{\mathbb{P}^{r}}(l)\right|_{C_{0}}\right) \cong H^{0}\left(\mathbb{P}^{1}, \mathcal{O}\left(d_{0} l\right)\right) \rightarrow H^{0}\left(\mathbb{P}^{1}, \mathcal{O}(d l)\right),
$$

where $C_{0} \cong \mathbb{P}^{1}$ is the parameterized component of $C$. The first map is simply the restriction and the last map is defined by multiplying a factor $\prod_{m=1}^{s}\left(a_{m} z_{0}-b_{m} z_{1}\right)^{l d_{m}}$, where $\left(a_{m}: b_{m}\right)$ are the nodal points on the parameterized $\mathbb{P}^{1}$ and $\left(z_{0}: z_{1}\right)$ the homogeneous coordinates on parameterized $\mathbb{P}^{1}$. It follows that $\sigma_{0}$ drops ranks only on the comb strata of types $\mu$ described in the previous section. Any hairy comb substrata obtained from $\mu$ by further degenerating the unparameterized components will not further reduce the rank of $\sigma_{0}$. Moreover, it has the following transversality property: $\sigma_{0}$ has generic corank $n_{1}$ and $n_{2}$ along $D_{\mu_{1}}$ and $D_{\mu_{2}}$ respectively. Then $\sigma_{0}$ has generic corank $n_{1}+n_{2}$ along the intersection of two strata.

The above fibrewise description actually holds globally. Namely, on the strata $D_{\mu}, \sigma_{0}$ is the following composition

$$
\varphi_{\mu}^{*} \sigma_{0}: \varphi_{\mu}^{*} \mathcal{E}_{d} \rightarrow p_{0}^{*} \mathcal{E}_{d_{0}} \stackrel{p_{0}^{*} \sigma_{0}}{\rightarrow} p_{0}^{*} \mathcal{F}_{d_{0}} \rightarrow \varphi_{\mu}^{*} \mathcal{F}_{d},
$$

where $p_{0}: D_{\mu} \rightarrow \bar{G}_{0, s}\left(\mathbb{P}^{r}, d_{0}\right)$ is the projection (see (9)), $\mathcal{E}_{d_{0}}$ and $\mathcal{F}_{d_{0}}$ on $\bar{G}_{0, s}\left(\mathbb{P}^{r}, d_{0}\right)$ are defined in Corollary 4 . The last map in 19 is the push-forward (along the first $\left.\mathbb{P}^{1}\right)$ of the following map

$$
\mathcal{O}_{\mathbb{P}^{1} \times\left(\mathbb{P}^{1}\right)^{s} \times \mathbb{P}_{d_{0}}^{r}}\left(d_{0} l, 0, \cdots, 0, l\right) \hookrightarrow \mathcal{O}_{\mathbb{P}^{1} \times\left(\mathbb{P}^{1}\right)^{s} \times \mathbb{P}_{d_{0}}^{r}}\left(d l, d_{1} l, \cdots, d_{s} l, l\right) .
$$

One can now apply the above study of degeneration type of $\sigma_{0}$ to the graph construction. Since the rank of $\sigma_{0}$ decreases only on the strata $D_{\mu}$, (17) becomes

$$
c_{\text {top }}\left(\mathcal{E}_{d}\right)=u^{*} \prod_{k=0}^{d l}(l H+k \hbar)+\sum_{\mu} m_{\mu} S_{\mu} .
$$

Namely, the only $Z_{\delta}$ in (17) are $D_{\mu}$ and $S_{\mu}=\eta_{\mu_{*}} c_{\text {top }}(\zeta)$. To write down $S_{\mu}$ explicitly in terms of characteristic classes in $\left.\sigma_{0}\right|_{D_{\mu}}: \varphi_{\mu}^{*} \mathcal{E}_{d} \rightarrow \varphi_{\mu}^{*} \mathcal{F}_{d}$ one would need a filtration of the above setting. Let us first deal with the simplest case when $\mu=\left(d_{0}, d_{1}\right)$, i.e. $D_{\mu}$ is a divisor. The universal curve on $D_{\mu}=\bar{G}_{0,1}\left(\mathbb{P}^{r}, d_{0}\right) \times_{\mathbb{P} r}$ $\bar{M}_{0,1}\left(\mathbb{P}^{r}, d_{1}\right)$ (generically) consists of one parameterized and one unparameterized components. The kernel of $\sigma_{0}$ can be identified with $p_{1}^{*} \mathcal{E}_{d_{1}}^{1}$, where $p_{1}: D_{\mu} \rightarrow$ $\bar{M}_{0,1}\left(\mathbb{P}^{r}, d_{1}\right)$ and $\mathcal{E}_{d_{1}}^{1}$ is the kernel of the evaluation morphism $e$ of bundles on $\bar{M}_{0,1}\left(\mathbb{P}^{r}, d_{1}\right)$

$$
0 \rightarrow \mathcal{E}_{d_{1}}^{1} \rightarrow \pi_{2 *} \mathrm{ev}_{2}^{*} \mathcal{O}_{\mathbb{P}^{r}}(l) \stackrel{e}{\rightarrow} \mathrm{ev}_{1}^{*} \mathcal{O}_{\mathbb{P}^{r}}(l) \rightarrow 0,
$$

where ev $2: \bar{M}_{0,2}\left(\mathbb{P}^{r}, d_{1}\right) \rightarrow \mathbb{P}^{r}$ and $\pi_{2}: \bar{M}_{0,2}\left(\mathbb{P}^{r}, d_{1}\right) \rightarrow \bar{M}_{0,1}\left(\mathbb{P}^{r}, d_{1}\right)$ forgets the second marked point. The kernel $\mathcal{E}_{d_{1}}^{1}$ can be further filtered by the order of zeros of $e$ :

$$
0=p_{1}^{*} \mathcal{E}_{d_{1}}^{d_{1} l+1} \subset p_{1}^{*} \mathcal{E}_{d_{1}}^{d_{1} l} \subset \cdots \subset p_{1}^{*} \mathcal{E}_{d_{1}}^{1} \subset \varphi_{\mu}^{*} \mathcal{E}_{d}
$$


where $\mathcal{E}_{d_{1}}^{k}$ consists of those sections of $\operatorname{ev}_{2}^{*} \mathcal{O}_{\mathbb{P}^{r}}(l)$ which vanishes at least to the $k$-th order at the marking. Similarly we can filter $\varphi_{\mu}^{*} \mathcal{F}_{d}$ by the span of the image of $\left.\sigma_{0}\right|_{k D_{\mu}}$ on $\mathbb{P}^{1} \times \mathbb{P}_{d_{0}}^{r}$ :

$$
\mathcal{F}_{d_{0}}^{k}:=H^{0}\left(\mathbb{P}^{1}, \mathcal{O}\left(d_{0} l+k-1\right)\right) \otimes \mathcal{O}_{\mathbb{P}^{1} \times \mathbb{P}_{d_{0}}^{r}}(k-1, l)
$$

such that

$$
p_{1}^{*} \mathcal{F}_{d_{0}}=u_{\mu}^{*} \mathcal{F}_{d_{0}}^{1} \subset \cdots \subset u_{\mu}^{*} \mathcal{F}_{\mu}^{d_{1} l} \subset \varphi_{\mu}^{*} \mathcal{F}_{d}
$$

is a filtration of $\varphi_{\mu}^{*} \mathcal{F}_{d}$ on $D_{\mu}$. Now for each $D_{\mu}$ there are $d_{1} l$ components $V_{\mu}^{k} \subset W_{\infty}$ $\left(\eta_{\mu}: V_{\mu} \rightarrow D_{\mu}\right)$ because the infinitesimal property of $\sigma_{0}$ along $D_{\mu}$. Each of $V_{\mu}^{k}$ is a birational image of a $\mathbb{P}^{1}$-bundle over $D_{\mu}$. By a local computation $\left([\sqrt[3]{3}), V_{\mu}^{k}\right.$ has multiplicity $k$, and the tautological bundle on $V_{\mu}^{k}$ can be expressed in terms of the filtration of $\mathcal{E}_{d}$ and $\mathcal{F}_{d}$ :

$$
0 \rightarrow p_{0}^{*} u_{\mu}^{*} \mathcal{F}_{d_{0}}^{k} \oplus p_{1}^{*} \mathcal{E}_{d_{1}}^{k+1} \rightarrow \zeta_{V_{\mu}^{k}} \rightarrow \mathcal{O}(-1) \rightarrow 0 .
$$

The contribution $S_{\mu}$ from the boundary strata $\mu=\left(d_{0}, d_{1}\right)$ can be therefore written as

$$
S_{\mu}=\varphi_{\mu_{*}}\left(e_{\mu} \cup c_{\mathrm{top}}\left(u_{\mu}^{*} \mathcal{F}_{d_{0}}^{1}\right)\right)=\varphi_{\mu_{*}}\left(e_{\mu} \cup u_{\mu}^{*} \prod_{k=0}^{d_{0} l}(l H+k \hbar)\right)
$$

where

$$
e_{\mu}=\sum_{k=1}^{d_{1} l}(-k) p_{d_{1}}^{*} c_{\mathrm{top}}\left(\mathcal{E}_{d_{0}}^{k+1}\right) \cup u_{\mu}^{*} c_{\mathrm{top}}\left(\mathcal{F}_{d_{0}}^{k} / \mathcal{F}_{d_{0}}^{1}\right) .
$$

This is exactly what we are looking for.

When $\mu$ is not a divisorial stratum we may use the above transversality property (of $\sigma_{0}$ concerning the intersection of two boundary strata). Since every $D_{\mu}$ is the intersection of divisors, and the corank of $\sigma_{0}$ at intersection is equal to the sum of the coranks generically, we can then obtain $S_{\mu}$ for general $\mu$ inductively. Let $\mu_{1}=\left(d_{0}, d_{1}\right)$ and $V_{\mu_{1}}^{k} \subset W_{\infty}$ be the $\mathbb{P}^{1}$-bundle over $D_{\mu_{1}}$. Now apply graph construction to the following vector bundle morphism on (pulled back to) $V_{\mu_{1}}^{k}$

$$
\varphi_{\mu}^{*} \mathcal{E}_{d} / p_{1}^{*} \mathcal{E}_{d_{1}}^{1} \cong p_{0}^{*} \mathcal{E}_{d_{0}} \stackrel{p_{0}^{*} \sigma_{0}}{\rightarrow} p_{0}^{*} \mathcal{F}_{d_{0}}=u_{\mu}^{*} \mathcal{F}_{d_{0}}^{1} .
$$

(Some obvious pull-backs will be omitted.) Then the components of $W_{\infty}^{\prime}$ over $V_{\mu_{1}}^{k}$ obtained from this construction map birationally to the components of $W_{\infty}$ over $\bar{G}_{0,0}\left(\mathbb{P}^{r}, d\right)$. For example, components of $V_{\mu^{\prime}}^{m} \subset W_{\infty}^{\prime}$ corresponding to boundary strata $\mu^{\prime}=\left(d_{0}-d^{\prime}, d^{\prime}\right)$ of $\bar{G}_{0,1}\left(\mathbb{P}^{r}, d_{0}\right)$ map to the components of $V_{\mu}^{m} \subset W_{\infty}$ over the boundary strata $\mu=\left(d_{0}-d^{\prime}, d^{\prime}, d_{1}\right)$ on $\bar{G}_{0,0}\left(\mathbb{P}^{r}, d\right)$. This implies that $V_{\mu}$ for any $\mu=\left(d_{0}, d_{1}, \cdots, d_{s}\right)$ with a fixed ordering of $d_{1}, \cdots, d_{s}, V_{\mu}^{k}$ are (birationally) towers of $\mathbb{P}^{1}$-bundles over $D_{\mu}$ (by first doing $\mu_{1}=\left(d_{0}+\cdots+d_{s-1}, d_{s}\right)$ then $\mu_{2}=$ $\left(d_{0}+\cdots+d_{s-2}, d_{s-1}, d_{s}\right)$, etc.). An explicit expression of $e_{\mu}$ can therefore be obtained:

$$
e_{\mu}=\prod_{m=1}^{s} \sum_{k_{m}=1}^{d_{m} l}\left(-k_{m}\right) p_{d_{m}}^{*} c_{\mathrm{top}}\left(\mathcal{E}_{d_{m}}^{k_{m}+1}\right) \cup u_{\mu}^{*} c_{\mathrm{top}}\left(\mathcal{F}_{\Sigma_{m}}^{k_{m}} / \mathcal{F}_{\Sigma_{m}}^{1}\right)
$$

\footnotetext{
${ }^{6}$ The explicit multiplicity is actually irrelevant to our result.
} 
where $\Sigma_{m}:=\sum_{a=0}^{m-1} d_{a}$ and $\mathcal{E}_{d_{m}}^{k}$ is defined to be the filtration of kernel on the $m$-th unparameterized component, similar to that defined in (22) and $\mathcal{F}_{\Sigma_{m}}^{1} \hookrightarrow \mathcal{F}_{\Sigma_{m}}^{k_{m}}$ is the push-forward of the inclusion of line bundles on $\mathbb{P}^{1} \times\left(\mathbb{P}^{1}\right)^{s} \times P_{d_{0}}$ to $\left(\mathbb{P}^{1}\right)^{s} \times P_{d_{0}}$ :

$$
\begin{aligned}
& \mathcal{O}\left(\sum_{a=1}^{m-1} d_{a} l, d_{1} l, \cdots, d_{m-1} l, 0, \cdots, 0, l\right) \\
\rightarrow & \mathcal{O}\left(\sum_{a=1}^{m-1} d_{a} l+k_{m}-1, d_{1} l, \cdots, d_{i-1} l, k_{m}-1,0, \cdots, 0, l\right) .
\end{aligned}
$$

This completes our proof of the convex case.

II. (Concave case) The proof of the concave case can in general be carried out in a similar way. However, some crucial modifications will be necessary. Now $\mathcal{E}_{d}=$ $R^{1} \pi_{*} \mathrm{ev}^{*} \mathcal{O}_{\mathbb{P}^{r}}(l)$ and $\mathcal{F}_{d}=H^{1}\left(\mathbb{P}^{1}, \mathcal{O}(d l)\right) \otimes u^{*} \mathcal{O}_{\mathbb{P}_{d}^{r}}(l)$ (with $l$ negative). Corollary 3 guarantees that there is a bundle map $\sigma_{1}$ between $\mathcal{F}_{d}$ and $\mathcal{E}_{d}$. We may apply Serre duality and find $\sigma_{1}^{*}:\left(\mathcal{E}_{d}\right)^{*} \rightarrow\left(\mathcal{F}_{d}\right)^{*}$. Carry out the graph construction to $\sigma_{1}^{*}$. The equation (18) (on $\left.D_{\left(d_{0}, \cdots, d_{s}\right)}\right)$ should be replaced by

$$
\begin{aligned}
& H^{0}\left(C, \omega_{C} \otimes f^{*} L^{-1}\right) \rightarrow H^{0}\left(C_{0},\left.\omega_{C_{0}}\left(\chi_{1}+\cdots+\chi_{s}\right) \otimes f^{*} L^{-1}\right|_{C_{0}}\right) \\
& \cong H^{0}\left(\mathbb{P}^{1}, \omega_{\mathbb{P}^{1}}\left(-d_{0} l\right) \otimes \mathcal{O}\left(\chi_{1}+\cdots+\chi_{s}\right)\right) \rightarrow H^{0}\left(\mathbb{P}^{1}, \omega_{\mathbb{P}^{1}}(-d l)\right) .
\end{aligned}
$$

Here $\chi_{1}, \cdots, \chi_{s}$ are the nodal points on the parameterized $\mathbb{P}^{1}$ (see Corollary 3 ) and $\omega_{C}$ is the dualizing sheaf of $C$.

A similar modification to 20 should also take place:

$$
c_{\text {top }}\left(E_{d}\right)=u_{X}^{*} \prod_{k=\left\langle c_{1}(L), d\right\rangle+1}^{-1}(l H+k \hbar)+\sum_{\mu} m_{\mu} S_{\mu} .
$$

For the filtration of the kernel of $\sigma_{1}^{*}$, we may use the following exact sequence (see (21))

$$
0 \rightarrow \mathcal{E}_{d_{1}}^{1} \rightarrow \pi_{*}\left(\mathrm{ev}_{2}^{*} \mathcal{O}_{\mathbb{P}^{r}}(l) \otimes \omega\left(x_{1}\right)\right) \stackrel{\text { res }}{\rightarrow} \mathrm{ev}_{1}^{*} \mathcal{O}_{\mathbb{P}^{r}}(l) \rightarrow 0
$$

where res is the residue at $x_{1}$. Again we can further filter $\mathcal{E}_{d_{1}}^{1}$ by the order of zeros of res, (as did in (22)). Similar filtration can be defined on $\varphi_{\mu}^{*} \mathcal{F}_{d}$. Namely

$$
\begin{aligned}
\mathcal{F}_{d_{0}}^{k_{m}}= & \omega_{\mathbb{P}^{1}}\left(x_{1}+\cdots+x_{s}+\sum_{a=1}^{m-1} d_{a}(-l)+k_{m}-1\right) \\
& \otimes \mathcal{O}_{\left(\mathbb{P}^{1}\right)^{s} \times P_{d_{0}}}\left(d_{1}(-l), \cdots, d_{m-1}(-l), k_{m}-1,0, \cdots, 0,(-l)\right) .
\end{aligned}
$$

Now a similar computation leads to

$$
\begin{aligned}
S_{\mu} & =\varphi_{\mu_{*}}\left(e_{\mu} \cup c_{\mathrm{top}}\left(u_{\mu}^{*} \mathcal{F}_{d_{0}}^{1}\right)\right) \\
& =\varphi_{\mu_{*}}\left(e_{\mu} \cup u_{\mu}^{*} \xi_{1} \cdots \xi_{s} \prod_{k=d_{0} l+1}^{-1}(l H+k \hbar)\right) .
\end{aligned}
$$

The rest is straightforward and is left to the reader. 


\section{Conclusion of the proofs}

4.1. Main contribution term. 7 Set $\mathrm{ev}=i \circ \mathrm{ev}_{X}$ and

$$
J_{X}^{E}(\beta)=J_{X}^{E}(\beta, \text { main })+\sum_{\nu} J_{X}^{E}(\beta, \nu) .
$$

In order to show that $\int_{X} J_{X}^{E} \simeq \int_{X} I_{X}^{E}$ it is sufficient to show $i_{*} J_{X}^{E} \simeq i_{*} I_{X}^{E}$ because

$$
\int_{X} e^{p t} \omega=\int_{\mathbb{P}^{r}} e^{h t} i_{*} \omega
$$

by the projection formula.

Recall that our goal is to compute $\mathrm{ev}_{*}^{\mathrm{vir}} \frac{c_{\text {top }}\left(E_{\beta}\right)}{\hbar(\hbar-\psi)}$. From (11) we have

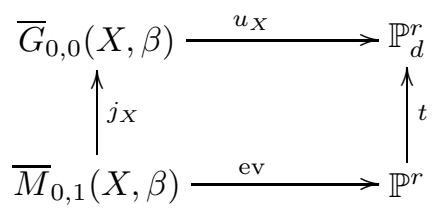

We can easily get, by correspondence of residues (12), the "main term":

$$
\begin{aligned}
& i_{*} J_{X}^{E}(\beta, \text { main })=\operatorname{ev}_{*}^{\operatorname{vir}}\left(\frac{j_{X}^{*} u_{X}^{*} \prod_{j} \prod_{k_{j}}\left(l_{j} H+k_{j} \hbar\right)}{\hbar(\hbar-\psi)}\right) \\
& =\frac{t^{*} u_{X *}^{\mathrm{vir}} u_{X}^{*} \prod_{j} \prod_{k_{j}}\left(l_{j} H+k_{j} \hbar\right)}{e\left(N_{\mathbb{P}^{r} \mid \mathbb{P}_{d}^{r}}\right)} \\
& =\frac{t^{*} u_{X *}^{\mathrm{vir}} 1}{e\left(N_{\mathbb{P}^{r} \mid \mathbb{P}_{d}^{r}}\right)} \cup t^{*} \prod_{j} \prod_{k_{j}}\left(l_{j} H+k_{j} \hbar\right) \\
& =\mathrm{ev}_{*}^{\mathrm{vir}}\left(\frac{1}{\hbar(\hbar-\psi)}\right) \cup \prod_{j} \prod_{k_{j}}\left(l_{j} h+k_{j} \hbar\right) \\
& =i_{*} I_{X}^{E} \text {. }
\end{aligned}
$$

Thus $I_{X}^{E}$ is really the main term of $J_{X}^{E}$. We will see that the boundary terms are of special forms and can be taken care of by a change of variables due to the non-negativity condition (on the tangent bundles of complete intersection $Y$ in $X$ ) stated in Theorem 1 .

4.2. Boundary terms and dimension counting. Before we start our discussion, we should remark that the term "dimension" here means virtual dimension.

${ }^{7}$ Here instead of going through the diagrams (25) 26, it might be possible to proceed by another (equivalent) way using Givental's double construction formula [11], which reads

$$
\mathcal{G}:=\sum_{\beta} q^{\beta} \int_{\left[\bar{G}_{0,0}(X, \beta)\right]^{\mathrm{vir}}} e^{P t} c_{\mathrm{top}}\left(E_{\beta}\right)=\int_{X} J_{X}^{E}\left(q e^{\hbar t}, \hbar\right) e^{p t} J_{X}^{E}(q,-\hbar),
$$

where $P=u_{X}^{*}\left(\mathcal{O}_{\mathbb{P}_{d}^{r}}(1)\right)$. This should give us $J_{X}^{E}\left(q, \hbar, t_{0}, t\right)$ (see (36) from formulas in Theorem 
Consider the commutative diagram (see (11)

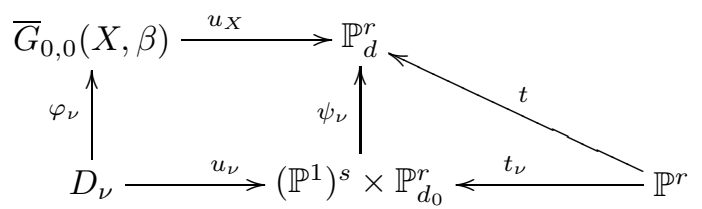

In the convex case (see (16)),

$$
\begin{aligned}
& i_{*} J_{X}^{E}(\beta, \nu) \\
= & \operatorname{ev}_{*}^{\operatorname{vir}}\left(\frac{j_{X}^{*} \sum_{\nu} \varphi_{\nu *} \frac{1}{s !}\left(e_{\nu} \cup u_{\nu}^{*} \prod_{j} \prod_{k_{j}=0}^{d_{0} l_{j}}\left(l_{j} H+k_{j} \hbar\right) \cap\left[D_{\nu}\right]^{\mathrm{vir}}\right)}{\hbar(\hbar-\psi)}\right) \\
= & \frac{t^{*} \mathrm{PD} u_{X *}\left(\sum_{\nu} \varphi_{\nu *} \frac{1}{s !}\left(e_{\nu} \cup u_{\nu}^{*} \prod_{j} \prod_{k_{j}=0}^{d_{0} l_{j}}\left(l_{j} H+k \hbar\right)\right) \cap\left[\bar{G}_{0,0}\right]^{\mathrm{vir}}\right)}{\prod_{k=1}^{d}(h+k \hbar)^{r+1}} \\
= & \frac{t^{*} \sum_{\nu} \frac{1}{s !} \psi_{\nu *}\left(u_{\nu}^{\mathrm{vir}} e_{\nu} \cup \prod_{j} \prod_{k_{j}=0}^{d_{0} l_{j}}\left(l_{j} H+k \hbar\right)\right)}{\prod_{k=1}^{d}(h+k \hbar)^{r+1}} .
\end{aligned}
$$

Here we have used (13) and the left square of the above commutative diagram (27).

Similar results holds in concave case: 8

$$
\begin{aligned}
& i_{*} J_{X}^{E}(\beta, \nu) \\
= & \operatorname{ev}_{P}^{\operatorname{vir}} *\left(\frac{j_{X}^{*} \sum_{\nu} \varphi_{\nu *}\left(e_{\nu} \cup u_{\nu}^{*} \prod_{j} \prod_{m=1}^{s} \xi_{m} \prod_{k_{j}=d_{0} l_{j}+1}^{-1}\left(l_{j} H+k \hbar\right) \cap\left[D_{\nu}\right]^{\mathrm{vir}}\right)}{\hbar(\hbar-\psi)}\right) \\
= & \frac{t^{*} \sum_{\nu} \frac{1}{s !} \psi_{\nu *}\left(u_{\nu}^{\mathrm{vir}} e_{\nu} \cup \prod_{j}\left(\xi_{1} \cdots \xi_{s} \prod_{k_{j}=d_{0} l_{j}}^{-1}(l H+k \hbar)\right)\right)}{\prod_{k=1}^{d}(h+k \hbar)^{r+1}} .
\end{aligned}
$$

Apply the correspondence of residues (12) to the right commuting triangle of (26)

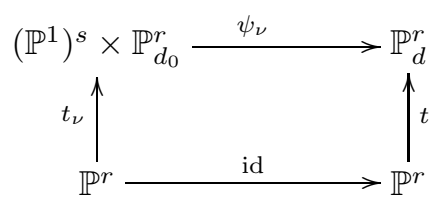

one has

$$
\begin{aligned}
& \frac{t^{*} \psi_{\nu_{*}} w}{\prod_{k=1}^{d}(h+k \hbar)^{r+1}} \\
& =\frac{t_{\nu}^{*} w}{\hbar^{s} \prod_{k=1}^{d_{0}}(h+k \hbar)^{r+1}},
\end{aligned}
$$

Then equation (27) becomes, by (28),

$$
i_{*} J_{X}^{E}(\beta, \nu)=\sum_{\nu} \frac{\prod_{j} \prod_{k=0}^{d_{0} l_{j}}\left(l_{j} h+k \hbar\right) \cup t_{\nu}^{*} u_{\nu}^{\mathrm{vir}} e_{*} e_{\nu}}{s ! \hbar^{s} \prod_{k=1}^{d_{0}}(h+k \hbar)^{r+1}} .
$$

\footnotetext{
${ }^{8}$ We have chosen to use small font for the discussion in the convex case in this subsection.
} 
Similarly in the concave case $\left(l_{j}<0\right)$

$$
i_{*} J_{X}^{E}(\beta, \nu)=\sum_{\nu} \frac{\prod_{j} \xi_{1} \cdots \xi_{s} \prod_{k=d_{0} l_{j}+1}^{-1}\left(l_{j} h+k \hbar\right) \cup t_{\nu}^{*} u_{\nu}^{\mathrm{vir}}{ }_{*} e_{\nu}}{s ! \hbar^{s} \prod_{k=1}^{d_{0}}(h+k \hbar)^{r+1}} .
$$

Therefore it remains to compute $u_{\nu}^{\mathrm{vir}} e_{\nu}$.

First let us work on the hypersurface case, i.e. $E=L$ and $L$ convex. Define $L_{\beta}^{k}:=i_{G}^{*}\left(\mathcal{E}_{d}^{k}\right)$ for the notational convenience. From Proposition 1

$$
e_{\nu}=\prod_{m=1}^{s} \sum_{k=1}^{d_{m} l}\left(-k_{m}\right) p_{\beta_{m}}^{*} c_{\mathrm{top}}\left(L_{\beta_{m}}^{k_{m}+1}\right) \cup u_{\nu}^{*} c_{\mathrm{top}}\left(\mathcal{F}_{\Sigma_{m}}^{k_{m}} / \mathcal{F}_{\Sigma_{m}}^{1}\right)
$$

where $\Sigma_{m}=d_{0}+d_{1}+\cdots+d_{m-1}$. Again, to simplify the notations, let us start with the divisorial strata, i.e. $\nu=\left(\beta_{0}, \beta_{1}\right)$. In this case,

$$
e_{\nu}=\sum_{k=1}^{d_{1} l}-(k) p_{\beta_{1}}^{*} c_{\mathrm{top}}\left(L_{\beta_{1}}^{k+1}\right) \cup u_{\nu}^{*} c_{\mathrm{top}}\left(\mathcal{F}_{\beta_{0}}^{k} / \mathcal{F}_{\beta_{0}}^{1}\right) \text {. }
$$

Therefore

$$
u_{\nu *}^{\mathrm{vir}} e_{\nu}=\sum_{k=1}^{d_{1} l}-(k) u_{\nu *}^{\mathrm{vir}} p_{\beta_{1}}^{*} c_{\mathrm{top}}\left(L_{\beta_{1}}^{k+1}\right) \cup c_{\mathrm{top}}\left(\mathcal{F}_{\beta_{0}}^{k} / \mathcal{F}_{\beta_{0}}^{1}\right) .
$$

It is easy to see that

$$
c_{\mathrm{top}}\left(\mathcal{F}_{\beta_{0}}^{k} / \mathcal{F}_{\beta_{0}}^{1}\right)=\prod_{m=1}^{k-1}\left(l\left(h+d_{0} \hbar\right)+m \xi\right)
$$

where $\xi$ is the equivariant point class of $\mathbb{P}^{1}$. Thus we only have to know $u_{\nu}^{\mathrm{vir}} p_{1}^{*} c_{\mathrm{top}}\left(L_{\beta_{1}}^{k+1}\right)$, which is in turn $\mathrm{PD} u_{X, 1_{*}} p_{0 *}\left(p_{1}^{*} c_{\text {top }}\left(L_{\beta_{1}}^{k+1}\right) \cap\left[D_{\nu}\right]^{\text {vir }}\right)$ because the morphism $u_{\nu}$ : $D_{\nu} \rightarrow \mathbb{P}^{1} \times \mathbb{P}_{d}^{r}$ factors through $p_{0}$

$$
D_{\nu} \stackrel{p_{0}}{\longrightarrow} G_{0,1}(X, \beta) \stackrel{u_{X}, 1}{\longrightarrow} \mathbb{P}^{1} \times \mathbb{P}_{d}^{r} .
$$

For this, consider the fibre square:

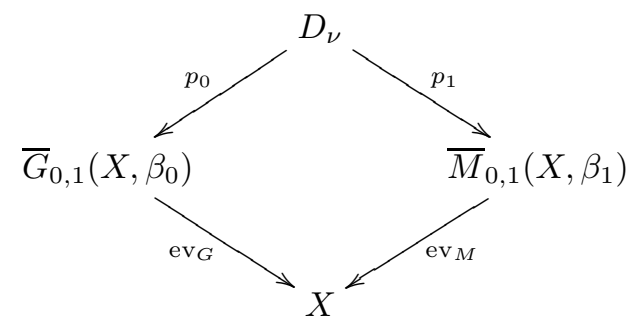

so that

$$
p_{0 *}\left(p_{1}^{*} c_{\mathrm{top}}\left(L_{\beta_{1}}^{k+1}\right) \cap\left[D_{\nu}\right]^{\mathrm{vir}}\right)=\left(\operatorname{ev}_{G}^{*} \mathrm{ev}_{M *}^{\mathrm{vir}} c_{\mathrm{top}}\left(L_{\beta_{1}}^{k+1}\right)\right) \cap\left[\bar{G}_{0,1}\left(X, \beta_{0}\right)\right]^{\mathrm{vir}} .
$$

However, by dimension counting, $\operatorname{ev}_{M *}^{\mathrm{vir}} c_{\mathrm{top}}\left(L_{\beta_{1}}^{k+1}\right)$ has the cohomological degree $2-k+\left\langle c_{1}(L)-c_{1}(X), \beta_{1}\right\rangle$, which is at most one for $k=1$ and at most zero for $k=2$. It vanishes otherwise. It is also obvious that if $\left\langle c_{1}(L)-c_{1}(X), \beta\right\rangle \leq-2$ for any $\beta$, i.e. the case Fano of index $\geq 2$, then all boundary contributions vanish. 
From our assumption that $i: X \rightarrow \mathbb{P}^{r}$ induces an isomorphism $i^{*}: N S\left(\mathbb{P}^{r}\right) \rightarrow$ $N S(X)$, the above degree one algebraic cohomology classes lies in the image of $i^{*}$ and we may conclude that

$$
\mathrm{ev}_{G}^{*} \mathrm{ev}_{M *}^{\mathrm{vir}} c_{\mathrm{top}}\left(L_{\beta_{1}}^{k+1}\right)=i_{G}^{*} \mathrm{ev}_{G_{0,1}\left(\mathbb{P}^{r}, d_{0}\right)}^{*}\left(c_{\nu}^{0}+c_{\nu}^{1} h\right)
$$

for $c^{i} \in \mathbb{Q}$. However, as shown in Lemma 1 that

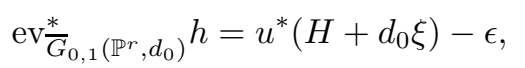

where $\epsilon$ is the exceptional divisor of $u$. Summing up, the boundary term for the strata $\nu=\left(\beta_{0}, \beta_{1}\right)$ is (see (29))

$$
\begin{aligned}
& i_{*} J_{X}^{L}(\beta, \nu)=\frac{\prod_{k=0}^{d_{0} l}(l h+k \hbar)}{\prod_{k=1}^{d}(h+k \hbar)} t_{\nu}^{*} u_{\nu *}^{\mathrm{vir}} e_{\nu} \\
= & i_{*}\left(J_{X}\left(\beta_{0}\right) \cup H_{\beta_{0}}^{L}\right) \cup \prod_{k=0}^{d_{0} l}\left(c_{\nu}^{0}\left(l\left(h+d_{0} \hbar\right)+\hbar\right)+c_{\nu}\left(h_{i}+d_{0}^{i} \hbar\right)\right) .
\end{aligned}
$$

Since $c^{0}, c^{i}$ are independent of $\beta_{0}$ from the above discussion, this proved the part (a) of the following proposition.

Proposition 2. Let $L$ be a convex line bundle on $X$ induced from $P$, and let $\lambda_{\nu}(p, \hbar)$ be defined by the following formula

$$
i_{*}\left(J_{X}\left(\beta_{0}\right) \cup H_{\beta_{0}}^{L} \cup \lambda_{\nu}(p, \hbar)\right):=\frac{\prod_{k=0}^{d_{0} l}(l h+k \hbar) \cup t_{\nu}^{*} u_{\nu}^{\mathrm{vir}} e_{\nu}}{\prod_{k=1}^{d_{0}}(h+k \hbar)^{r+1}},
$$

which is $i_{*} J_{X}^{L}(\beta, \nu)$.

(a) If $\nu=\left(\beta_{0}, \beta_{1}\right)$, then $\lambda_{\nu}(p, \hbar)$ is linear and satisfies

$$
\lambda_{\left(\beta_{0}, \beta_{1}\right)}(p, \hbar)=\lambda_{\left(0, \beta_{1}\right)}\left(p+d_{0} \hbar, \hbar\right) .
$$

(b) for $\nu=\left(\beta_{0}, \beta_{1}, \cdots, \beta_{s}\right)$, we have

$$
\lambda_{\nu}(p, \hbar)=\lambda_{\left(\Sigma_{1}, \beta_{1}\right)}(p, \hbar) \lambda_{\left(\Sigma_{2}, \beta_{2}\right)}(p, \hbar) \cdots \lambda_{\left(\Sigma_{s}, \beta_{s}\right)}(p, \hbar)
$$

where $\Sigma_{m}=\sum_{a<m} \beta_{a}$.

Proof. (of part (b)) The equation (34) requires only to compute $t_{\nu}^{*} u_{\nu *} e_{\nu}$, with $e_{\nu}$ described by (29)

$$
u_{\nu *} e_{\nu}=\prod_{m} \sum_{k_{m}}\left(-k_{m}\right) u_{\nu *} p_{\beta_{m}}^{*} c_{\text {top }}\left(L_{\beta_{m}}^{k_{m}+1}\right) \cup c_{\text {top }}\left(\mathcal{F}_{\Sigma_{m}}^{k_{m}} / \mathcal{F}_{\Sigma_{m}}^{1}\right)
$$

Using the same argument to the following diagram

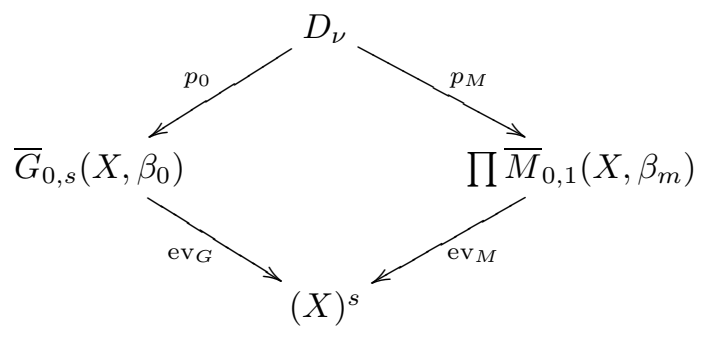


one obtains that

$$
c_{\text {top }}\left(\mathcal{F}_{\Sigma_{m}}^{k_{m}} / \mathcal{F}_{\Sigma_{m}}^{1}\right)=\prod_{a=1}^{k_{m}-1}\left(l h+l \Sigma_{m} \hbar+\sum_{b=1}^{m-1} l d_{b} \xi_{b}+a \xi_{m}\right) .
$$

Dimension counting and (32) gives us the proof of part (b).

In the concave case a similar modification goes through.

Proposition 3. Let $L$ be a concave line bundle and let $\lambda_{\nu}$ be defined as in (33). Then part (a) and (b) hold. Furthermore, $\lambda_{\nu}$ depends only on $\hbar$.

Proof. From (30) for $J_{P}^{L}(\beta, \nu)$, we only have to compute $u_{\nu}^{\mathrm{vir}}{ }_{*} e_{\nu}$. A straightforward modification, by fibre square (31) and dimension counting, will lead to the conclusion that only degree zero (constant) terms survives in $\operatorname{ev}_{M *}^{\mathrm{vir}} c_{\text {top }}\left(L_{\beta_{1}}^{k+1}\right)$. The difference is that the cohomological degree of $\mathrm{ev}_{M *}^{\mathrm{vir}} c_{\mathrm{top}}\left(L_{\beta_{1}}^{k+1}\right)$ is only $-k+\left\langle-c_{1}(L)-c_{1}(X), \beta_{1}\right\rangle$, because the rank of $L^{k+1}$ in this case is one less than that of $L^{k+1}$ in convex case.

Therefore $\lambda_{\nu}=$ constant $\cdot t_{\nu}^{*} \prod_{m=1}^{s} \xi_{m}=$ constant $\cdot \hbar^{s}$. This allows us to set $\lambda_{\left(\beta_{0}, \beta_{1}\right)}=$ constant $\cdot \hbar$.

Corollary 5. The same is true when the rank of $E$ is greater than 1.

The proof uses the inductive property of $e_{\nu}$ and is completely analogous to the above argument.

Summarizing the above discussion, we have

\section{Theorem 6.}

$$
i_{*} J_{X}^{E}=i_{*} \sum_{\beta} q^{\beta} \sum_{\nu} \frac{J_{X}\left(\beta_{0}\right) \cup H_{\beta_{0}}^{E} \cup \prod_{m=1}^{s} \lambda_{\beta_{m}}\left(p+\Sigma_{m} \hbar, \hbar\right)}{s ! \hbar^{s}}
$$

such that $\lambda_{\beta_{m}}(p, \hbar)$ are linear form in $p, \hbar$ and is independent of the total degree $\beta$ in the background.

Proof. Set $\lambda_{\beta_{m}}(p, \hbar)=\lambda_{\left(0, \beta_{m}\right)}(p, \hbar)$ and apply Propositions 2 and 3 .

4.3. Mirror transformation. In this subsection we will omit the push-forward symbol $i_{*}$. All equalities are assumed to hold after pushing-forward to $\mathbb{P}^{r}$.

Now we are ready to find a change of variables (mirror transformation) between $J_{X}^{E}$ and $I_{X}^{E}$. Note that we will use $\beta$ both as an element in $H_{2}(X)$ and a number. In the case $\operatorname{Pic}(X)=1$, this should not cause confusion.

$$
\begin{aligned}
& t_{0} \mapsto t_{0}+f_{0}(q) \hbar, \\
& t \mapsto t+f_{1}(q),
\end{aligned}
$$

where $f_{0}(q), f_{1}(q)$ are formal power series in $q$. After the change of variables, $e^{\left(t_{0}+p t\right) / \hbar} I_{X}^{E}(q, \hbar)$ becomes

$$
e^{f_{0}(q)+\frac{1}{\hbar} p f_{1}(q)} e^{\frac{1}{\hbar}\left(t_{0}+p t\right)} I_{X}^{E}\left(q e^{f_{1}(q)}, \hbar\right),
$$

which we would like to equate to $J_{X}^{E}(q, \hbar)$. 
Let us set $f_{1}(q)=\sum_{\beta} a_{\beta} q^{\beta}$ and $f_{0}(q)=\sum_{\beta} b_{\beta} q^{\beta}$. Then (37) can be expanded as:

$$
\begin{aligned}
& e^{f_{0}(q)+\frac{1}{\hbar} h f_{1}(q)} e^{\frac{1}{\hbar}\left(t_{0}+p t\right)} I_{X}^{E}\left(q e^{f_{1}(q)}, \hbar\right) \\
= & \sum_{\beta_{0}} q^{\beta_{0}} e^{f_{0}(q)+\left(\frac{p}{\hbar}+\beta_{0}\right) f_{1}(q)} I_{X}^{E}\left(\beta_{0}\right) \\
= & \sum_{\beta} q^{\beta} \sum_{\sum_{m=0}^{s} \beta_{m}=\beta} \frac{1}{s !} I_{X}^{E}\left(\beta_{0}\right) \prod_{m=1}^{s}\left(b_{\beta_{m}}+a_{\beta_{m}}\left(\beta_{0}+\frac{p}{\hbar}\right)\right) .
\end{aligned}
$$

In order to prove Theorem 1 we need to find $a_{\beta}, b_{\beta}$ so that (38) is equal to $J_{X}^{E}(q, \hbar)$. The following simple lemma in [3] is useful:

Lemma 2. Let

$$
Q(q)=\sum_{\beta} q^{\beta} \sum_{\sum_{m=1}^{s} \beta_{m}=\beta, d_{m} \neq 0} \frac{1}{s !} \prod_{m=0}^{s}\left(y_{\beta_{m}}+x_{\beta_{m}} B_{m-1}\right),
$$

where $B_{m-1}:=\sum_{k=1}^{m-1} \beta_{k},\left(B_{0}=0\right)$. Then $\log (Q(q))$ is a linear function of $y_{\beta}$.

Corollary 6. Use the notation from the above lemma. Define $z_{\beta}$ by

$$
Q(q)=\exp \left(\sum_{\beta \neq 0} z_{\beta} q^{\beta}\right) .
$$

Then

$$
z_{\beta}=\sum_{\sum_{m=1}^{s} \beta_{m}=\beta} \frac{1}{s !} y_{\beta_{1}} \prod_{m=1}^{s} x_{\beta_{m}} B_{m-1}
$$

Proof. Expand equations (40) and (39). The $q^{\beta}$ term is

$$
\sum_{\sum_{m=1}^{s} \beta_{m}=\beta} \frac{1}{s !} \prod_{m=1}^{s} z_{\beta_{m}}=\sum_{\sum_{m=1}^{s} \beta_{m}=\beta} \frac{1}{s !} \prod_{m=1}^{s}\left(y_{\beta_{m}}+x_{\beta_{m}} B_{m-1}\right) .
$$

By Lemma 2 the right hand side of $(42)$ is linear with respect to $y_{\beta}$. The linear in $y_{\beta}$ term on the RHS is exactly (41).

Back to the proof of Theorem 1. We wish to equate (36) to (38). Set $\lambda_{\beta}=$ $c_{\beta}^{0} \hbar+c_{\beta}^{1} p$, then (36) can be written as

$$
\begin{aligned}
J_{X}^{E}(q) & =\sum_{\beta_{0}} \sum_{\sum_{m=1}^{s} \beta_{m}^{\prime}=\beta^{\prime}} q^{\beta^{\prime}+\beta_{0}} J_{X}\left(\beta_{0}\right)\left(\prod_{j} H_{\beta_{0}}^{L_{j}}\right) \\
& \frac{1}{s !} \prod_{m=1}^{s}\left(\left(c_{\beta_{m}^{\prime}}^{0}+c_{\beta_{m}^{\prime}}^{1}\left(\frac{p}{\hbar}+\beta_{0}\right)\right)+c_{\beta_{m}^{\prime}}^{1} B_{m-1}\right) \\
= & \sum_{\beta_{0}} q^{\beta_{0}} J_{X}\left(\beta_{0}\right)\left(\prod_{j} H_{\beta_{0}}^{L_{j}}\right) \\
& \sum_{\sum_{m=1}^{s} \beta_{m}^{\prime}=\beta^{\prime}} q^{\beta^{\prime}} \frac{1}{s !} \prod_{m=1}^{s}\left(\left(c_{\beta_{m}^{\prime}}^{0}+c_{\beta_{m}^{\prime}}^{1}\left(\frac{p}{\hbar}+\beta_{0}\right)\right)+c_{\beta_{m}^{\prime}}^{1} B_{m-1}\right) .
\end{aligned}
$$


Apply Corollary 6, we find a new variables $z_{\beta^{\prime}}$ such that $z_{\beta^{\prime}}$ are linear in $c_{\beta_{m}^{\prime}}^{0}+$ $c_{\beta_{m}^{\prime}}^{1}\left(\frac{p}{\hbar}+\beta_{0}\right)$ and polynomial in $c_{\beta_{j}^{\prime}}^{1}$. Therefore there are constants $b_{\beta^{\prime}}, a_{\beta^{\prime}}$ such that $z_{\beta^{\prime}}=b_{\beta^{\prime}}+a_{\beta^{\prime}}\left(\frac{p}{\hbar}+\beta_{0}\right)$, and $b_{\beta^{\prime}}, a_{\beta^{\prime}}$ are independent of $\beta_{0}$. This implies that

$$
J_{X}^{E}(q)=\sum_{\sum_{m=0}^{s} \beta_{m}=\beta} q^{\beta} I_{X}^{E}\left(\beta_{0}\right) \frac{1}{s !} \prod_{m=1}^{s}\left(b_{\beta_{m}}+a_{\beta_{m}}\left(\frac{p}{\hbar}+\beta_{0}\right)\right),
$$

which is exactly (38). One can easily see from the above proof that the case $E$ being a direct sum of convex and concave bundles requires little modification. Our proof of the Theorem 1 is therefore complete.

4.4. Proof of Theorem 2. The proof of case 2 is very easy. As we have seen in $\S 4.2$ that the use of fibre square (31) (35) and dimension counting guarantees that $u_{\nu *}^{\text {vir }} e_{\nu}=0$. Therefore $J_{X}^{E}(\beta, \nu)=0$.

The proof of case 1 goes a slightly different way. f. As we have seen in the proof of Proposition 3 that $u_{\nu}^{\text {vir }} e_{\nu}$ contains only constant terms. Therefore the boundary contribution should come from

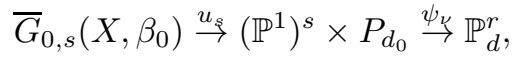

and should be of the form

$$
\psi_{\nu *}\left((\text { constant })\left(u_{s *} 1\right) \cup \prod_{j=1}^{\mathrm{rk}(E)} \xi_{1} \cdots \xi_{s} \prod_{k_{j}=\left\langle c_{1}\left(L_{j}\right), \beta_{0}\right\rangle+1}^{-1}\left(l_{j} H+k_{j} \hbar\right)\right),
$$

which has cohomological dimension greater than $J_{X}^{E}(\beta$, main). Therefore constant $=$ 0 .

The proof of direct sum case is the combination of the above arguments.

4.5. Proof of Corollary 2. To simplify our notations, set

$$
J^{\prime}:=i_{*} c_{\mathrm{top}}(E) e^{\frac{1}{\hbar}\left(t_{0}+p t\right)} J_{X}^{E}(q, \hbar)
$$

and

$$
J_{\vee}^{\prime}:=i_{*} \frac{1}{c_{\mathrm{top}}(E)} e^{\frac{1}{\hbar}\left(t_{0}+p t\right)} J_{X}^{E^{\vee}}(q, \hbar) .
$$

Notice that $J_{\vee}^{\prime}$ is actually well defined as $J_{X}^{E^{\vee}}(q, \hbar)$ always has a factor $c_{\text {top }}\left(E^{\vee}\right)$. More precisely, consider the exact sequence

$$
0 \rightarrow R^{0} \pi_{*}\left(\mathrm{ev}^{*}\left(E^{\vee}\right) \otimes \mathcal{O}\left(-x_{1}\right)\right) \rightarrow R^{0} \pi_{*} \mathrm{ev}^{*}\left(E^{\vee}\right) \rightarrow \mathrm{ev}^{*} E^{\vee} \rightarrow 0 .
$$

Then in fact

$$
\begin{aligned}
J_{\vee}^{\prime}= & i_{*} e^{\frac{1}{\hbar}\left(t_{0}+p t\right)} \sum_{\beta} q^{\beta} \operatorname{ev}_{*}^{\operatorname{vir}}\left(\frac{c_{\text {top }}\left(R^{0} \pi_{*}\left(\mathrm{ev}^{*}\left(E^{\vee}\right) \otimes \mathcal{O}\left(-x_{1}\right)\right)\right)}{\hbar(\hbar-\psi)}\right) \\
= & i_{*} e^{\frac{1}{\hbar}\left(t_{0}+p t\right)} J_{X}^{E^{\vee}} \frac{1}{c_{\text {top }}\left(E^{\vee}\right)} .
\end{aligned}
$$

Similar interpretation holds for $J^{\prime}$ : it is the $J$-function of the bundle $R^{1} \pi_{*}\left(\mathrm{ev}^{*}(E) \otimes\right.$ $\left.\mathcal{O}\left(-x_{1}\right)\right)$, multiplied by $e^{\frac{1}{\hbar}\left(t_{0}+p t\right)}$. Notice that the ranks of the two bundles $R^{0} \pi_{*}\left(\operatorname{ev}^{*}\left(E^{\vee}\right) \otimes\right.$ $\left.\mathcal{O}\left(-x_{1}\right)\right)$ and $R^{1} \pi_{*}\left(\mathrm{ev}^{*}(E) \otimes \mathcal{O}\left(-x_{1}\right)\right)$ are the same.

\footnotetext{
${ }^{9}$ It is also possible to prove the convex case in this way.
} 
It follows from Theorems 11 and 2

$$
J^{\prime}=I^{\prime}:=e^{\frac{1}{\hbar}\left(t_{0}+p t\right)} \sum_{\beta} q^{\beta} J_{X}(\beta) \cup \prod_{j} \prod_{k_{j}=1}^{\left\langle c_{1}\left(L_{j}\right), \beta\right\rangle}\left(c_{1}(L)+k \hbar\right)
$$

and

$$
J_{\vee}^{\prime} \sim I_{\vee}^{\prime}:=(-1)^{\mathrm{rk}(E)} e^{\frac{1}{\hbar}\left(t_{0}+p t\right)} \sum_{\beta} q^{\beta} J_{X}(\beta) \cup \prod_{j} \prod_{k_{j}=\left\langle c_{1}\left(L_{j}\right), \beta\right\rangle+1}^{0}\left(c_{1}(L)+k \hbar\right) .
$$

Notice the change of limits in the products of $k$ due to $c_{\text {top }}(E)$ and $c_{\text {top }}\left(E^{\vee}\right)$ and the possible sign coming from the ratio of $c_{\text {top }}(E)$ and $c_{\text {top }}\left(E^{\vee}\right)$.

The difference between $I^{\prime}$ and $I_{\vee}^{\prime}$ can be easily figured out by using the quantum differential equation [11.

Claim. There is a power series $\phi(q)$ such that

$$
\phi(q) I^{\prime}=I_{\vee}^{\prime}
$$

We now sketch the proof of (43). Fix a basis $\left\{e_{a}\right\}$ of the vector space $H^{*}(X)$ with $e_{1}=1$. Let

$$
S_{a b}:=\sum_{\beta} q^{\beta}\left(e_{a}, e^{\frac{1}{\hbar}\left(t_{0}+p t\right)} \frac{e_{b}}{\hbar-\psi}\right)_{0,2, \beta}
$$

be a matrix of genus zero two-point Gromov-Witten invariants via the "fundamental class" $c_{\text {top }}\left(E_{\beta}\right) \cup c_{\text {top }}(E) \cap\left[\bar{M}_{0,2}(X, \beta)\right]^{\text {vir }}$. Note that we have identified $t_{i}=\log q_{i}$. Givental's theory of quantum differential equation says

$$
\hbar \frac{\partial}{\partial t_{i}} S=p_{i} \circ S
$$

where $p_{i}$ ○ is the quantum multiplication matrix. By the (virtual) dimension counting $J_{X}$ is a polynomial in $\hbar^{-1}$ of the form $1+O\left(\hbar^{-\left\langle c_{1}(E), \beta\right\rangle}\right)$. Therefore

$$
I^{\prime}=J^{\prime}=\left(1+O\left(\hbar^{-\operatorname{rk}(E)}\right)\right) e^{\frac{1}{\hbar}\left(t_{0}+p t\right)} .
$$

Notice that $S_{1 a}=\left\langle J^{\prime}, e_{a}\right\rangle$. This implies that the first row $S_{1 a}$ of $S$ has the same order in $\hbar^{-1}$.

Set $v_{j}:=c_{1}\left(L_{j}\right)$ and $\partial_{j}$ be the directional derivative on the direction $v_{j}$. First of all $\left(\prod_{j=1}^{\mathrm{rk}(E)} \partial_{j}\right)\left(I^{\prime} / c_{\mathrm{top}}(E)\right)=I_{\vee}^{\prime}$ by simple derivations. Now apply induction on the order $m$ of the differential operator $\mathcal{D}_{m}:=\prod_{j=1}^{m} \hbar \partial_{j}$. I claim that

$$
\mathcal{D}_{m}(S)=\left(\prod_{j=1}^{m} v_{j}\right) \circ S
$$

for $m<\operatorname{rk}(E)$.

The case $m=1$ is true by (44). Suppose that (45) is true for some $m<$ $\operatorname{rk}(E)-1$. This implies that $\mathcal{D}_{m} S=\left(\prod_{j}^{m} v\right) \circ+O\left(\hbar^{-1}\right)$. Therefore $\left(\left(\prod_{j}^{m} v\right) \circ\right)_{1 a}$ are independent of $t$ from LHS. Now differentiate one more time:

$$
\mathcal{D}_{m+1} S=\left(\hbar \partial_{m+1}\left(\prod_{j=1}^{m} v_{j}\right) \circ S+\left(\left(\prod_{j=1}^{m} v_{j}\right) \circ v_{m+1} \circ\right) S .\right.
$$


and the first row of RHS will be $\left\langle\left(\prod_{j=1}^{m} v_{j}\right) \circ v_{m+1}, e_{a}\right\rangle+O\left(\hbar^{-1}\right)$. Because the first row of the LHS modulo $\hbar^{-1}$ is independent of $t$, we have again $\left(\prod_{j=1}^{m} v_{j}\right) \circ v_{m+1}=$ $\prod_{j=1}^{m+1} v_{j}$. Thus (45) holds. If $m=\operatorname{rk}(E)-1$, then the first row of LHS of 446) would be certain power series $\phi(q)$ of $q\left(\bmod \hbar^{-1}\right)$. Since the first row of first term on RHS still vanishes $\left(\bmod \hbar^{-1}\right)$, we have the first row of (46) equal to

$$
\begin{aligned}
\phi(q) I^{\prime} & =\phi(q)\left(\prod_{j=1}^{\mathrm{rk}(E)} v_{j}\right)\left(\frac{I^{\prime}}{c_{\mathrm{top}}(E)}\right) \\
& =\left(\prod_{j=1}^{\mathrm{rk}(E)}\left(v_{j} \circ\right)\right) I^{\prime}=\mathcal{D}_{\mathrm{rk}(E)} I^{\prime} \\
& = \pm I_{\vee}^{\prime} .
\end{aligned}
$$

\section{REFERENCES}

[1] K. Behrend, Gromov-Witten invariants in algebraic geometry, Invent. Math. 127 (1997), no. 3, 601-617.

[2] K. Behrend, B. Fantechi, The intrinsic normal cone, Invent. math. 128 (1997), no. 1, 45-88.

[3] A. Bertram. Another way to enumerate rational curves with torus actions, math.AG/9905159.

[4] G. Bini, C. de Concini, M. Polito, C. Procesi, On the work of Givental relative to mirror symmetry, Appunti dei Corsi Tenuti da Docenti della Scuola. [Notes of Courses Given by Teachers at the School] Scuola Normale Superiore, Pisa, 1998. ii+92 pp.

[5] P. Candelas, X. de la Ossa, P. Green, L. Parkes, A pair of Calabi-Yau manifolds as an exactly soluble superconformal theory, Nuclear Phys. B 359 (1991), no. 1, 21-74.

[6] D. A. Cox, S. Katz, Y.-P. Lee, Virtual Fundamental Classes of Zero Loci, to appear in Enumerative geometry in physics, Contemporary Mathematics, AMS. math.AG/0006116.

[7] W. Fulton, Intersection theory, Springer-Verlag, 1984.

[8] W. Fulton, R. Pandharipande, Notes on stable maps and quantum cohomology, Algebraic geometry - Santa Cruz 1995, Proce. Symp. Pure. Math. 62. Part 2, (1997) 45-96.

[9] A. Gathmann, Absolute and relative Gromov-Witten invariants of very ample hypersurfaces, math.AG/9908054

[10] A. Gathmann, Relative Gromov-Witten invariants and the mirror formula, math.AG/0009190

[11] A. Givental, Equivariant Gromov-Witten invariants, IMRN 13 (1996) 613-663.

[12] A. Givental, Elliptic Gromov-Witten invariants and the generalized mirror conjecture, Integrable systems and algebraic geometry (Kobe/Kyoto, 1997), 107-155, World Sci. Publishing, River Edge, NJ, 1998.

[13] A. Givental, Topics in enumerative geometry, unpublished course notes, Berkeley, Fall 1997.

[14] T. Graber, R. Pandharipande, Localization of virtual classes, Invent. Math. 135 (1999), no. $2,487-518$.

[15] B. Kim, Quantum hyperplane section theorem for homogeneous spaces, Acta Math. 183 (1999), no. 1, 71-99.

[16] M. Kontsevich, Enumeration of rational curves via torus actions, The moduli space of curves (Texel Island, 1994), 335-368, Progr. Math., 129, Birkhäuser Boston, Boston, MA, 1995.

[17] Y.-P. Lee, R. Pandharipande, A reconstruction theorem in quantum cohomology and quantum $K$-theory, in preparation.

[18] J. Li, G. Tian, Virtual moduli cycles and Gromov-Witten invariants of algebraic varieties. J. Amer. Math. Soc. 11 (1998), no. 1, 119-174.

[19] B. Lian, K. Liu, S.-T. Yau, Mirror principle I, Asian J. Math. 1 (1997), no. 4, 729-763.

[20] B. Lian, K. Liu, S.-T. Yau, Mirror principle II, Sir Michael Atiyah: a great mathematician of the twentieth century. Asian J. Math. 3 (1999). no. 1, 109-146.

[21] B. Lian, K. Liu, S.-T. Yau, Mirror principle III, math.AG/9912038

[22] R. Pandharipande, Rational curves on hypersurfaces (after A. Givental), Séminaire Bourbaki. Vol. 1997/98. Astérisque No. 252, (1998), Exp. No. 848, 5, 307-340. 
[23] E. Witten, Phases of $N=2$ theories in two dimensions, Nuclear Phys. B 403 (1993), no. $1-2,159-222$.

Department of Mathematics, UCLA, Los Angeles, CA 90095-1555

E-mail address: yplee@math.ucla.edu 